\title{
Primary types in the collection of molluscs in the KwaZulu-Natal Museum: Patellogastropoda and Lepetellida
}

\author{
Igor V. Muratov'1,2, Elodie Heyns-Veale ${ }^{1,3}$ \\ I KwaZulu-Natal Museum, P. Bag 9070, Pietermaritzburg, 3200 South Africa 2 School of Life Sciences, Uni- \\ versity of KwaZulu-Natal, Scottsville, 3206, South Africa 3 South African Institute for Aquatic Biodiversity, \\ Somerset Street, Makhanda 6140, South Africa \\ Corresponding author: Igor V. Muratov (imuratov@nmsa.org.za) \\ Academic editor: D.G. Herbert | Received 13 March 2020 | Accepted 13 May 2020 | Published 1 July 2020 \\ http://zoobank.org/A507F6C1-EA79-46C0-940F-B0A62BD86A4D \\ Citation: Muratov IV, Heyns-Veale E (2020) Primary types in the collection of molluscs in the KwaZulu-Natal Museum: \\ Patellogastropoda and Lepetellida. African Invertebrates 61(1): 49-81. https://doi.org/10.3897/AfrInvertebr.61.51989
}

\begin{abstract}
All primary (name-bearing) types of Patelloidea, Lottioidea, Fissurelloidea and Scissurelloidea deposited in the KwaZulu-Natal Museum are presented. The reference to the original publication, including the original generic position, the type locality, the collector and the cited dimensions of the type specimen(s), is provided for each species, followed by information from the label for each type in the NMSA collection (type locality, collector and catalogue number), size of the type specimen, brief remarks and colour photographs.
\end{abstract}

\section{Keywords}

Fissurelloidea, Lottioidea, Mollusca, Patelloidea, Scissurelloidea

\section{Introduction}

All primary (name-bearing) types of Patellogastropoda (Patellidae and Lottiidae) and part of Vetigastropoda (Lepetellida: Fissurelloidea and Scissurelloidea) deposited in the KwaZulu-Natal Museum (NMSA) were annotated and illustrated here in accordance with the ICZN (International Code of Zoological Nomenclature) recommendation $72 \mathrm{~F} .4$, as the third part of the ongoing revision of the primary type material deposited in NMSA. The historical review of NMSA collection of molluscs can be found in our

Copyright I.V. Muratov \& E. Heyns-Veale. This is an open access article distributed under the terms of the Creative Commons Attribution License (CC BY 4.0), which permits unrestricted use, distribution, and reproduction in any medium, provided the original author and source are credited. 
first publication on the subject (see Muratov and Davis 2011), where we presented illustrated annotations on primary types of Scaphopoda and Cephalopoda. Second publication was on Polyplacophora (Muratov 2014).

Five shells of "Acmaea" albonotata E.A. Smith, 1901 (NMSA-MOL 01284/T525) from the type locality were labelled "syntypes" by A.C. Bruggen. However, after examination of the original description (E.A. Smith 1901: 107, Pl. I, figs 14, 16) and the original label, it became apparent that the shells deposited in NMSA were not seen by Smith. In addition, the shells in NMSA differ in shape, size and sculpture from the original description. Thus, since they are not types, they are not illustrated here.

The "neotype" of "Patella" obtecta Krauss, 1848: 47-48, Tab. III, fig. 11 (NMSAMOL 0D2159/T3291) was erroneously (ICZN 75.8) designated (Robson 1986: 313 (figs 20-23), 315) since syntypes still exist in Museum für Naturkunde, Berlin (ZMB/ Moll-28428) and in Museum of Comparative Zoology, Cambridge (MCZ: Mala: 152477/Acc 1173) and thus, is not illustrated here.

\section{Material and methods}

The collection of the primary types of Patelloidea, Lottioidea, Fissurelloidea and Scissurelloidea in the KwaZulu-Natal Museum consists of 19 holotypes, one syntype and one neotype, all collected along the eastern coastline of South Africa, from Sodwana Bay in the north to Port Alfred in the south.

The reference to the original publication, including the original generic position, the type locality, the collector and the cited dimensions of the primary type specimen(s), is provided for each species. This information on the original description is followed by the details from the label for each type in the NMSA collection (type locality, collector and catalogue number) and measured dimensions of the type illustrated in this publication. The types are empty dry shells and the number of specimens is one, if not stated otherwise. Types in NMSA historically were given two catalogue numbers: standard, the same as for non-types and an additional number with "T" prefix, to highlight its type status. All standard NMSA numbers are now given in the new format: with added "NMSA-MOL 0" prefix. However, in references to previous publications, all numbers are given in the historical format. Comprehensive labels of the types were not produced by authors of original descriptions, except for Diodora fuscocrenulata E.A. Smith, 1906. All standard museum labels were produced by collection managers after types were received from authors. All information given for each type was copied from the labels and hand-written catalogue, not from the original descriptions. Provincial names were standardised based on their current status. Comprehensive information on paratypes is given in addition, because original descriptions often lack some information from labels and catalogue books. Paratypes, however, were not illustrated here. Types in other collections were not verified unless they were required to clarify the status of types deposited in NMSA. The current status of each species is based here on the most recent comprehensive publication found and the complete synonymy is not given since this is not a taxonomic revision. 
All shells were measured under a stereomicroscope using an ocular micrometer, separately calibrated for each standard magnification against a Vernier Calliper. Shells larger than $10 \mathrm{~mm}$ were measured by the same Calliper. All scales were individually calculated for each illustrated shell and corresponding scales for each photograph were resized to match the illustrated scale. All shell sizes are given using the following template: $D \times s D \times H$, where $D$ is major diameter, $s D$ is smaller diameter $\left(90^{\circ}\right.$ to $\left.D\right)$ and $H$ is height. None of the shells were cleaned to avoid possible damage and none of the shells were damaged during preparation of this publication.

The following acronyms and abbreviations are used: ICZN - International Code of Zoological Nomenclature; MN - research vessel Meiring Naudé; n/d - no data; NHMUK - The Natural History Museum, London, UK (formerly known as BNHM and BMNH) and NMSA - The KwaZulu-Natal Museum, Pietermaritzburg, South Africa (formerly known as Natal Museum).

\section{List of primary types}

Class Gastropoda Cuvier, 1795

Subclass Patellogastropoda Lindberg, 1986

Superfamily Patelloidea Rafinesque, 1815

Family Patellidae Rafinesque, 1815

\section{Patella aphanes Robson, 1986}

aphanes Robson, 1986: 306, figs 1-3 (holotype), figs 4-11 (paratypes) [Patella, Hibberdene, Natal South Coast (G. Robson, 15 October 1985): 22.1×17.4×9.7 mm].

Material examined. Holotype. South Africa • KwaZulu-Natal, Hibberdene; 15 Oct. 1985; G. Robson leg.; on Perna perna, LST rocks; T3285; NMSA-MOL 0D2155 (soft parts in ethanol); Fig. $1(21.8 \times 17.3 \times 8.6 \mathrm{~mm})$.

Paratypes. South Africa • KwaZulu-Natal, Cape Vidal; 16 Nov. 1971; R. Kilburn leg.; T3324; NMSA-MOL 009440.

South Africa • KwaZulu-Natal, Mapelane; Nov. 1983; R. Kilburn leg.; T3325; NMSA-MOL 0B6945.

South Africa • KwaZulu-Natal, Chaka's Rock, intertidal zone, 24 Feb. 1985; D. Herbert leg.; on Perna perna; T3296; NMSA-MOL 0D0489.

South Africa • 2; KwaZulu-Natal, Reunion Rocks, intertidal zone; 7 Feb. 1985; R. Kilburn, D. Herbert, R. Fregona leg.; T3322; NMSA-MOL 0D0361.

South Africa $\bullet 7$ of 8; same data as holotype, figured (figs 4-11) in the original description; T3295; NMSA-MOL 0D2156.

South Africa 40 in ethanol; same data as holotype (mussel-dwelling); T3327; NMSA-MOL 0S2157.

South Africa 10 in ethanol; same data as holotype (Lithophyllum coated rocks); T3328; NMSA-MOL 0D2158. 


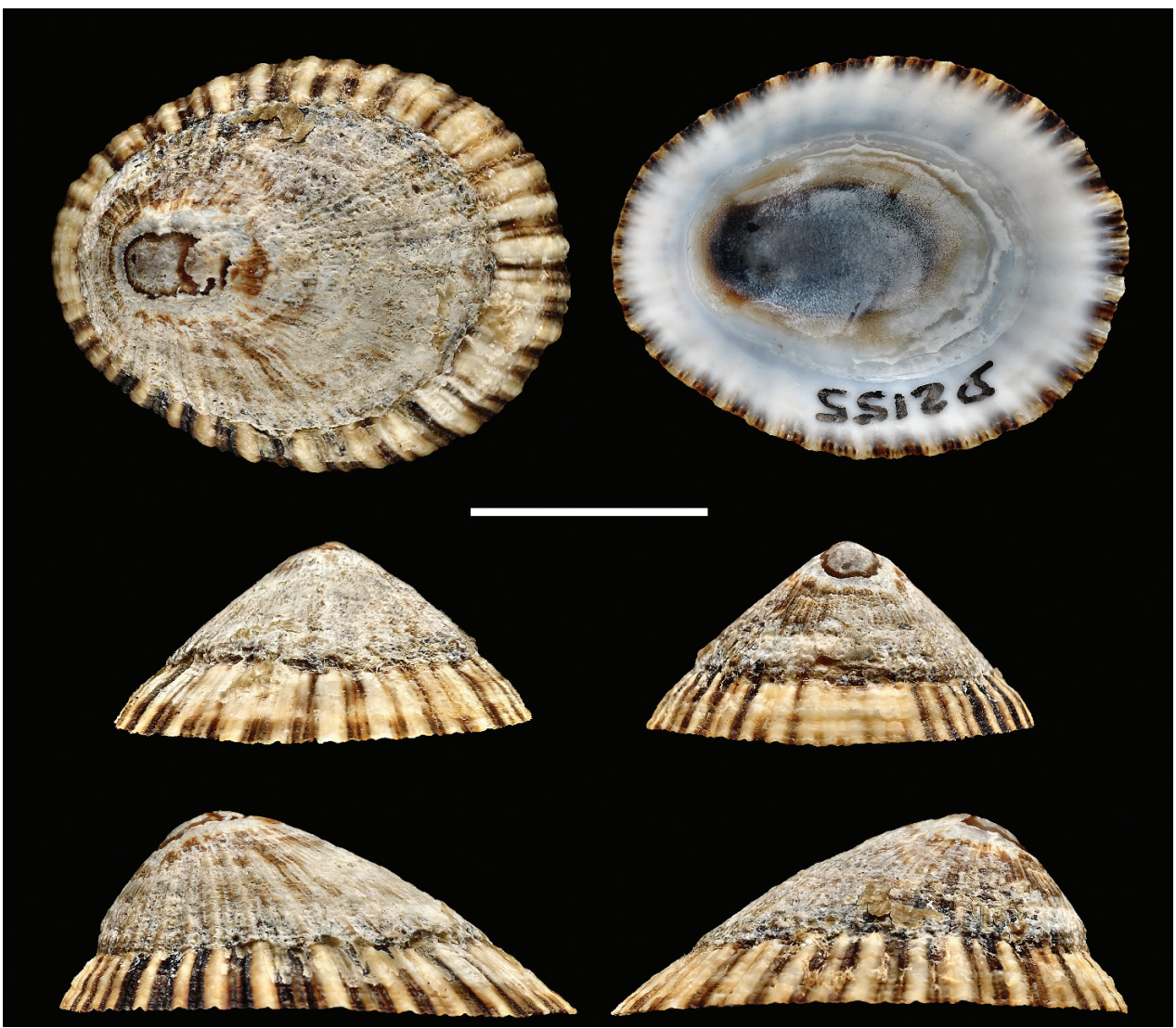

Figure I. Patella aphanes Robson, 1986. Holotype T3285/NMSA-MOL 0D2155. Scale bar: 10 mm.

South Africa - 2 of 3; KwaZulu-Natal, Port Edward; Jul. 1977; Marais leg.; T3286; NMSA-MOL 0A6281.

South Africa 14 of 18 in ethanol, one without soft parts; KwaZulu-Natal, Port Edward; 27-28 Aug. 1977; Marais leg.; on Perna; T4328; NMSA-MOL 0D2170.

South Africa • Eastern Cape, Lwandile/Mdumbi; Jul. 1981; R. Kilburn, R. Fregona leg.; T3326; NMSA-MOL 0C0148.

South Africa • 5; Eastern Cape, Coffee Bay; Y. McLellan leg.; ex Albany Mus. 1980; T3321; NMSA-MOL 0B6182.

South Africa • 4; Eastern Cape, Mkambati area, Mgwetyane R. mouth; R. Kilburn leg.; Aug. 1983; T3320; NMSA-MOL 0C5767.

South Africa • 3; Eastern Cape, Dwesa; May 1984; R. Kilburn leg.; Beach drift; T3323; NMSA-MOL 0C5909.

Current status. Scutellastra aphanes (Robson, 1986); Nakano and Ozawa (2007).

Remarks. There are 18 paratypes in ethanol mentioned in the original description under A6281/T3286. However, this number refers to 3 paratypes (T3286/NMSAMOL 0A6281), one of which was donated to Drivas (12 Oct. 1988), which are shells without soft parts, not in ethanol. There are 14 specimens in ethanol with the catalogue 
number NMSA-MOL 0D2170. Since these are the only other specimens of S. aphanes in ethanol, which in addition match the locality and collector as in the original description, they are assumed to be paratypes and were given new type number T4328 in preparation of this manuscript. There are another two shells in NMSA-MOL 0D489 paratype lot that were not chosen as paratypes. Prefix "C" was omitted in the original description for C5909. Figured paratypes were given wrong number (D2157/T3327 instead of D2156/T3295) in the original description (Robson 1986: figs 4-11). One (of originally eight paratypes: T3295/NMSA-MOL 0D2156) was sent in 1990 to Zoological Museum of Moscow University.

\section{Superfamily Lottioidea Gray, 1840} Family Lottiidae Gray, 1840

\section{Acmaea (Tectura) maraisi Kilburn, 1977}

maraisi R.N. Kilburn, 1977: 174, figs 1-3 (holotype) [Acmaea (Tectura), TRANSKEI: Mzamba (Fossil Head), near the Mtamvuna River mouth (J.P. Marais): $3.2 \times 3.0 \times 2.3 \mathrm{~mm}]$.

Material examined. Holotype. South Africa • Eastern Cape, Mzamba, beach drift; Apr. 1976; J.P. Marais leg.; don. J.P. Marais, 06 May 1976; T2051; NMSA-MOL 0A4585; Fig. $2(3.65 \times 2.96 \times 2.19 \mathrm{~mm})$.

Paratypes. South Africa - 10; same data as holotype; T2061; NMSAMOL 0A5051.

South Africa • Eastern Cape, Mzamba; May 1977; R. Kilburn leg.; T2148; NMSA-MOL 0A5848.

Current status. Asteracmea maraisi (Kilburn, 1977); WoRMS Editorial Board (2020).

Remarks. Generic position of this species remains uncertain. Its relation to Asteracmea has been suggested in the original description (Kilburn 1977: 175) but formal decision is awaiting proper revision of this group. Overexposed areas were intentionally introduced on Fig. 2 to show otherwise barely visible details of sculpture.

Subclass Vetigastropoda Salvini-Plawen, 1980

Order Lepetellida Moskalev, 1971

Superfamily Fissurelloidea J. Fleming, 1822

Family Fissurellidae J. Fleming, 1822

Subfamily Diodorinae Odhner, 1932

\section{Glyphis fuscocrenulata E.A. Smith, 1906}

fuscocrenulata E.A. Smith, 1906: 56-57, Pl VIII, fig. 6 [Glyphis, Port Shepstone and Umkomaas, Natal: $16 \times 11.25 \times 6 \mathrm{~mm}]$. 


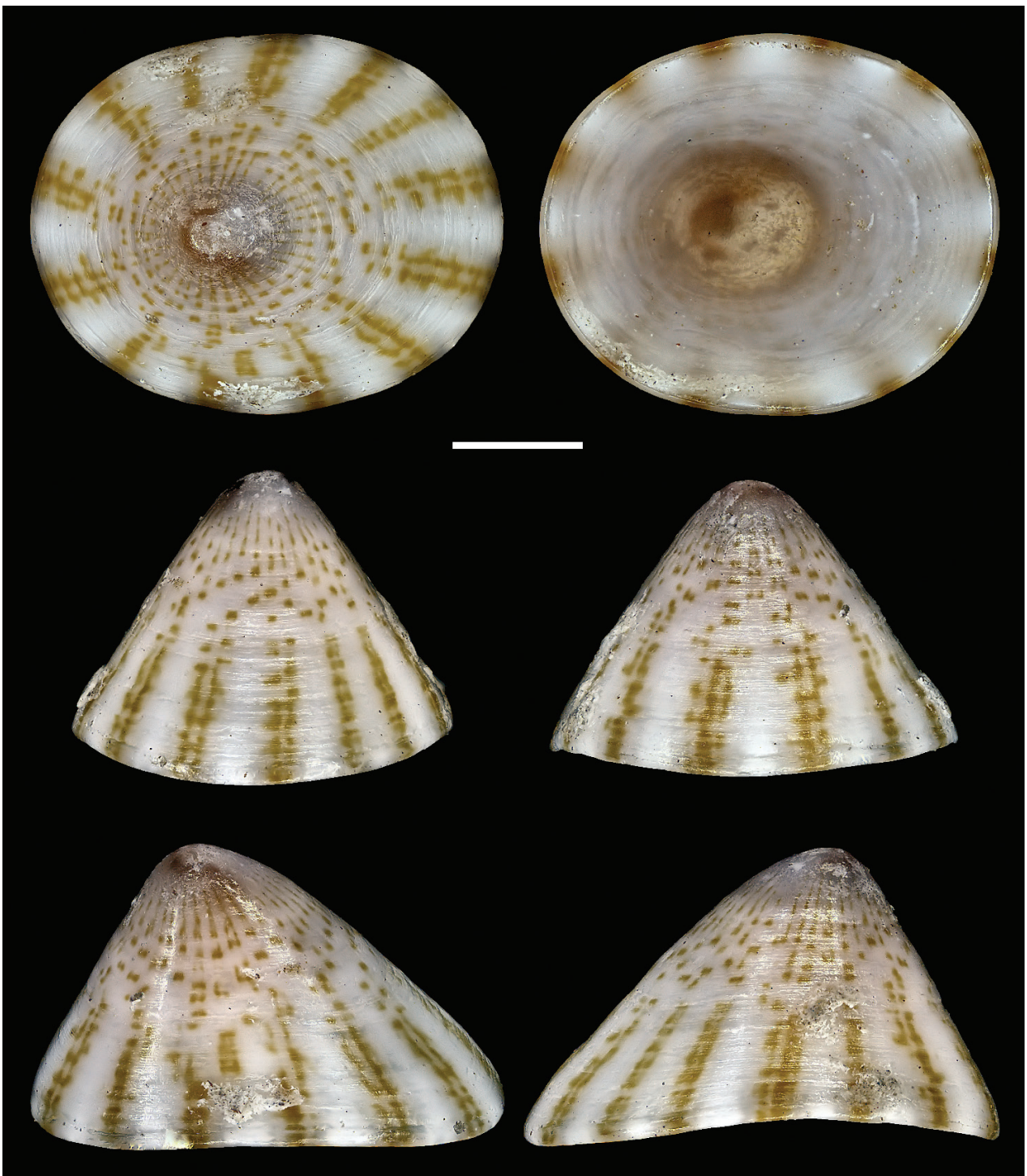

Figure 2. Acmaea (Tectura) maraisi Kilburn, 1977. Holotype T2051/NMSA-MOL 0A4585. Scale bar: $1 \mathrm{~mm}$.

Material examined. Syntype. South Africa - KwaZulu-Natal, Umkomaas; H.C. Burnup leg.; T524; NMSA-MOL 001270; Fig. $3(14.13 \times 9.65 \times 5$ mm).

Current status. Diodora fuscocrenulata (E. A. Smith, 1906); Herbert (2015).

Remarks. The shell (NMSA-MOL 001270) from Umkomaas and two of three shells (NHMUK: 1906.6.23.15-17) from Port Shepstone in the Natural History Museum (London, UK) were not explicitly mentioned in the original description. The sentence "However, the specimen described appears to be fairly mature" (Smith 1906: 


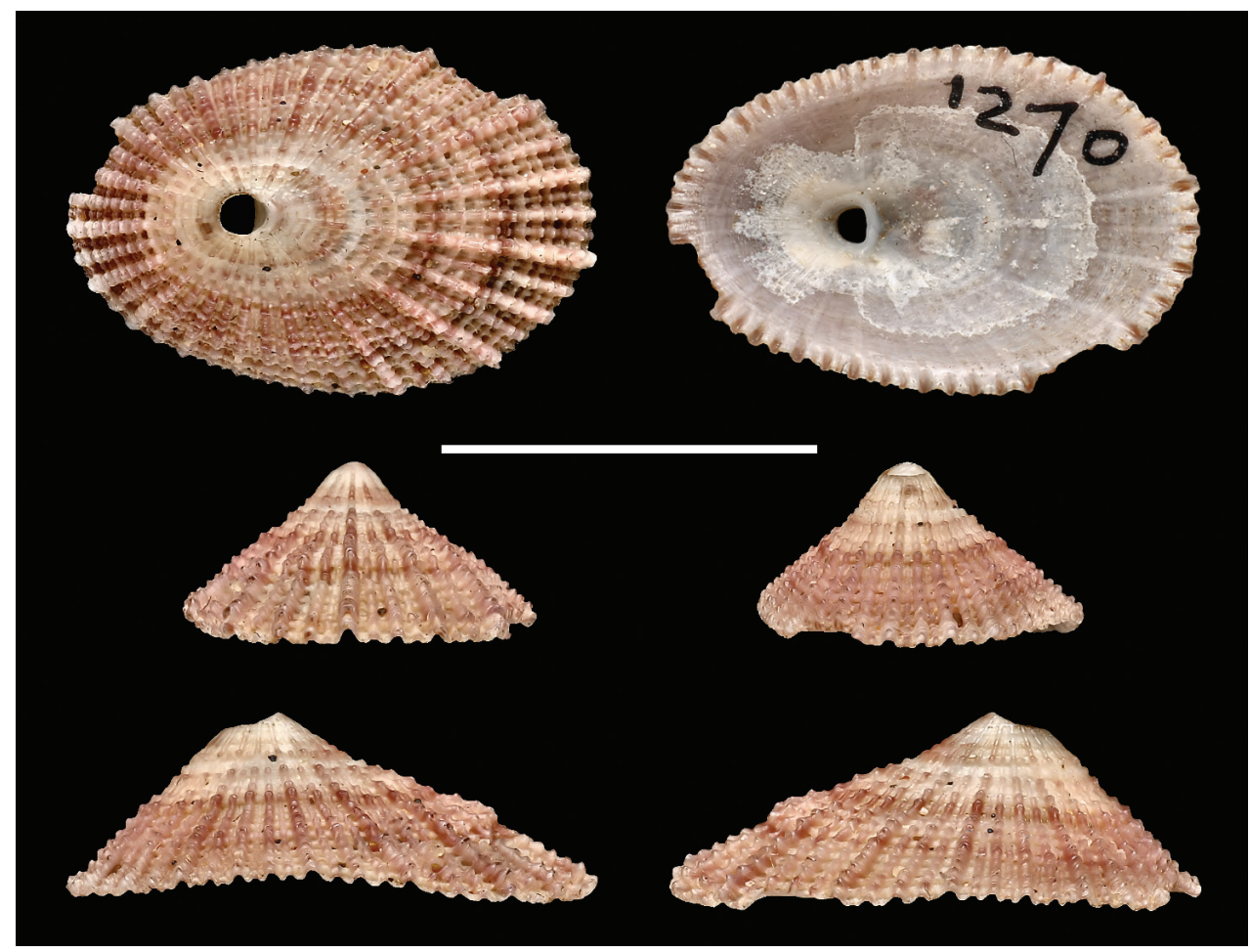

Figure 3. Glyphis fuscocrenulata E.A. Smith, 1906. Syntype T524/NMSA-MOL 001270. Scale bar: 10 mm.

57) implies that the description was based on one shell, which makes it holotype by monotypy (ICZN 73.1.2.). However, Smith was aware that the shell from Umkomaas belongs to the same species, as indicated in the description: "Hab. - Port Shepstone and Umkomaas, Natal" (Smith 1906: 57), which suggests that the shell from Umkomaas is part of the type series (see the Example for ICZN 72.4.1.1). We have not made changes in nomenclature and treat all four shells as syntypes, thereby not restricting possibilities in the next taxonomic revision.

\section{Diodora procurva Herbert, 1989}

procurva Herbert, 1989: 173, figs 1-3, 7 (holotype), 4-6 (paratype) [Diodora, off Mbashe River, Transkei (32 $\left.18.2^{\prime} \mathrm{S}, 2^{\circ} 04.1^{\prime} \mathrm{E}\right), 200-220 \mathrm{~m}$, sponge rubble. Dredged R.V. Meiring Naudé: $31.4 \times 21.8 \mathrm{~mm}$, height $21.7 \mathrm{~mm}$ ].

Material examined. Holotype. South Africa - Eastern Cape, off Mbashe River, $32^{\circ} 18.2^{\prime} \mathrm{S}, 29^{\circ} 04.1^{\prime} \mathrm{E}$, depth 200-220 m, station Q1; 18 Jul. 1982; dredged MN; sponge rubble; T124; NMSA-MOL 0E5938 (ex C1984); Fig. 4 (31.5×21.9×21.6 mm).

Paratypes. South Africa • 2; same data as holotype; T125; NMSA-MOL 0C1984. 


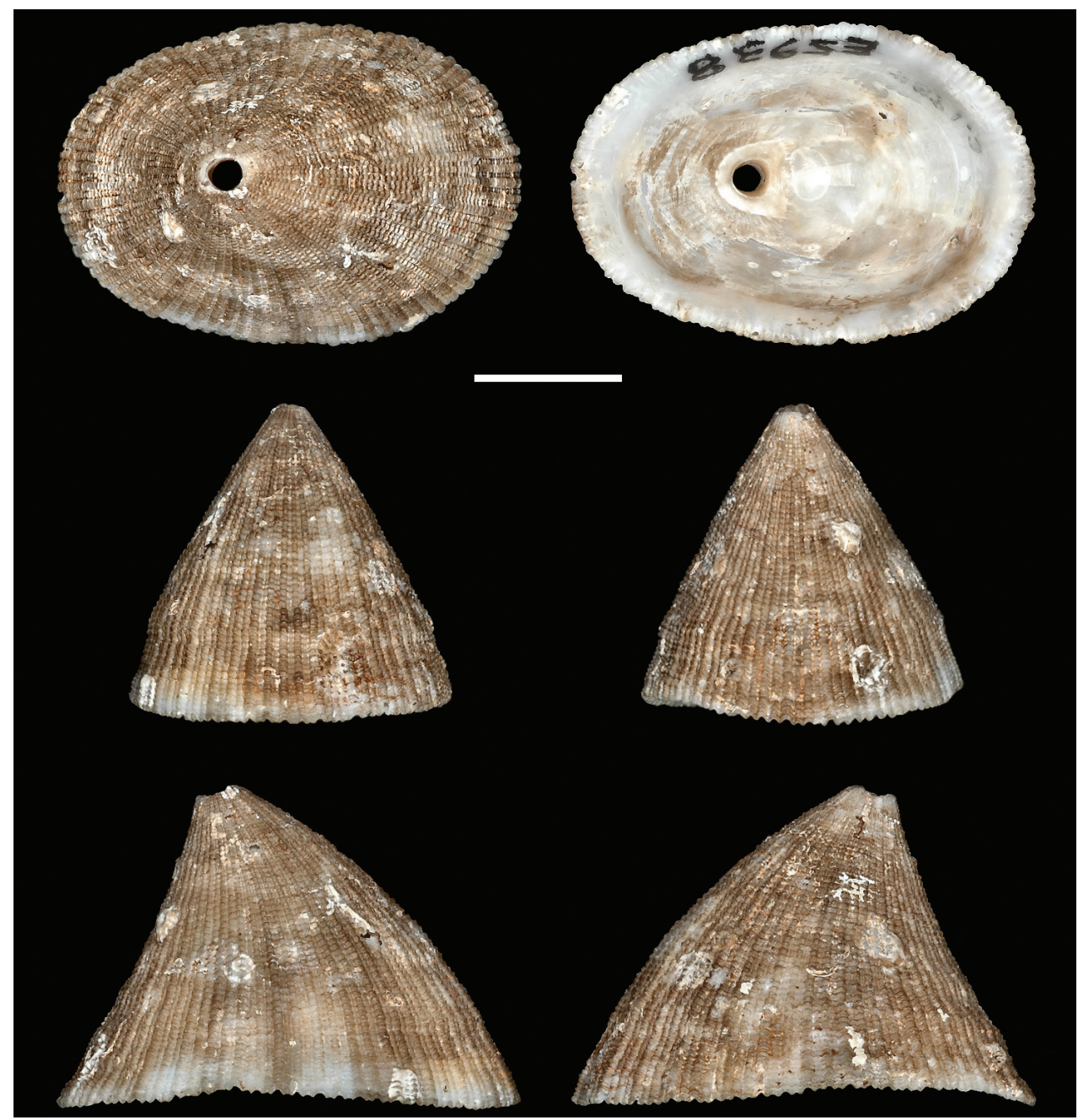

Figure 4. Diodora procurva Herbert, 1989. Holotype T124/NMSA-MOL 0E5938. Scale bar: 10 mm

South Africa • Eastern Cape, off Nqabara Point, 32²5.9'S, 2856.5'E, depth 130-150 m, station S7; 9 Jun 1983; dredged MN; sponges and stylasterids; T126; NMSA-MOL 0C5036.

South AfricA • Eastern Cape, off Qora River, 3255.6'S, 2849.0'E, depth 480-490 m, station U14; 14 Jul. 1984; dredged MN; sandy mud; T128; NMSA-MOL 0C6310.

South Africa - KwaZulu-Natal, S.E. of Sheffield Beach, 29³3.5'S, 3147.0'E, depth $180 \mathrm{~m}$, station XX135; 14 Jun. 1988; dredged MN; sponge rubble; T127; NMSA-MOL 0E4598. Figured (figs 4, 5) in the original description.

South Africa - 5; KwaZulu-Natal, S.E. of Neill Peak (Cunge), 2844.4'S, $32^{\circ} 32.2^{\prime} \mathrm{E}$, depth 320-340 m, station ZP5; 12 Jun. 1988; dredged MN; sandy mud; T129; NMSA-MOL 0E3970.

Current status. Diodora procurva Herbert, 1989; original combination. 


\section{Subfamily Emarginulinae Children, 1834}

\section{Emarginula connelli Kilburn, 1978}

connelli Kilburn, 1978: 437-439, Pl. 4b, f [Emarginula, off Sodwana Bay, Zululand in $100 \mathrm{~m}$ (ex C.S.I.R. Water Research): $6.9 \times 4.8 \times 5.2 \mathrm{~mm}$ ].

Material examined. Holotype. South Africa • KwaZulu-Natal, Sodwana Bay, depth 100 m, station G3; ex C.S.I.R. Water Research; T2199; NMSA-MOL 0A5762; Fig. 5 $(6.84 \times 4.83 \times 4.99 \mathrm{~mm})$.

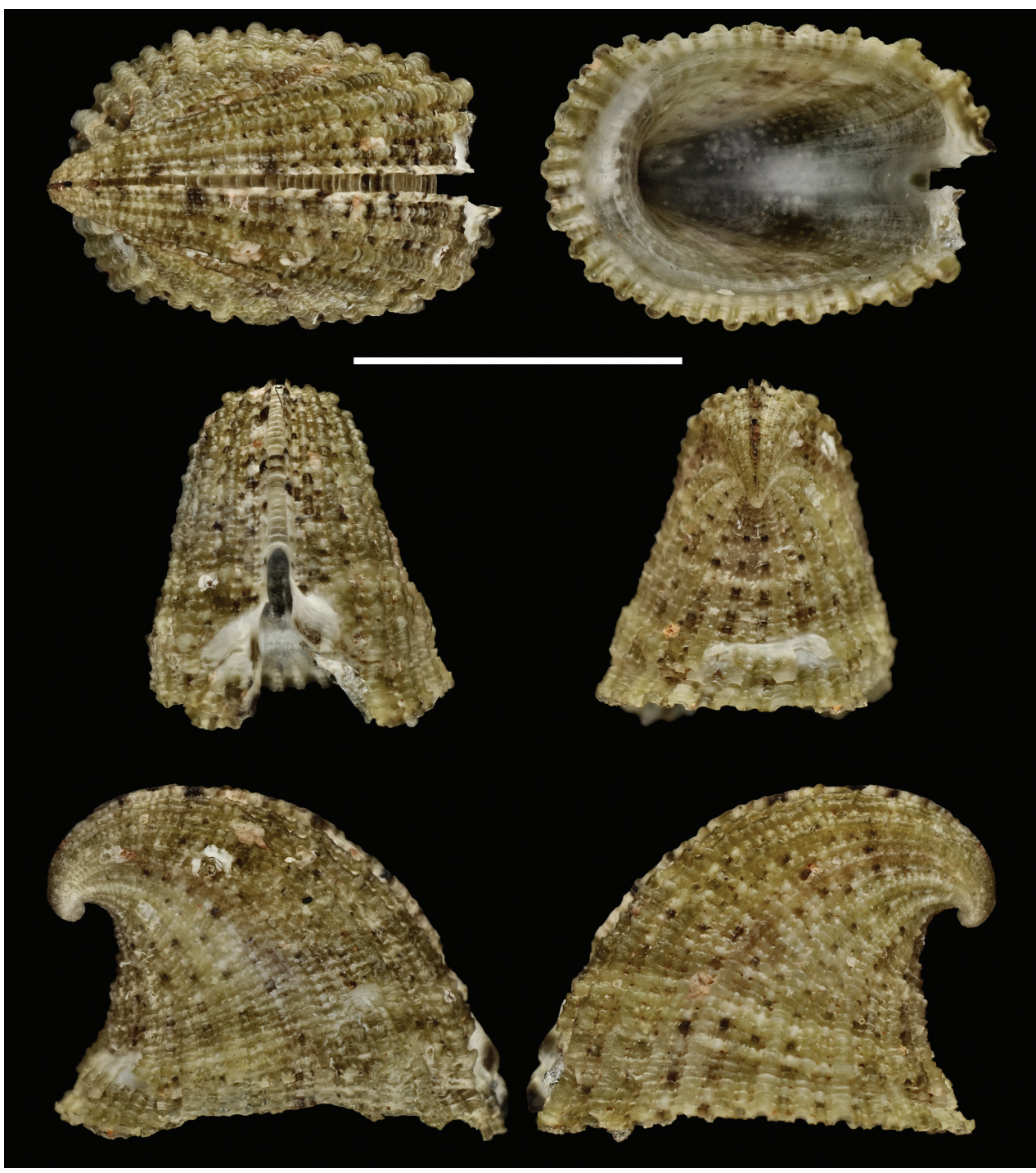

Figure 5. Emarginula connelli Kilburn, 1978. Holotype T2199/NMSA-MOL 0A5762. Scale bar: 5 mm. 
Current status. Emarginula connelli Kilburn, 1978; original combination.

Remarks. Was known in 1978 only from the type locality. However, one paratype of Emarginula thorektes Kilburn, 1978 (T2192/NMSA-MOL 0A5764) from Port Edward (see below) is Emarginula connelli Kilburn, 1978.

\section{Emarginula koon Kilburn, 1978}

koon Kilburn, 1978: 439-440, Pl. 5, 10 [Emarginula, Shelly Beach, near Port Shepstone (don. R. Cock): $18.0 \times 12.9 \times 7.9 \mathrm{~mm}]$.

Material examined. Holotype. South Africa - KwaZulu-Natal, Shelly Beach, near Port Shepstone; don. R. Cock, Nov. 1975; T2196; NMSA-MOL 0B0147; Fig. 6 $(18 \times 12.9 \times 8 \mathrm{~mm})$.

Paratypes. South Africa - 2; Same data as holotype; T2195; NMSAMOL 0A3724.

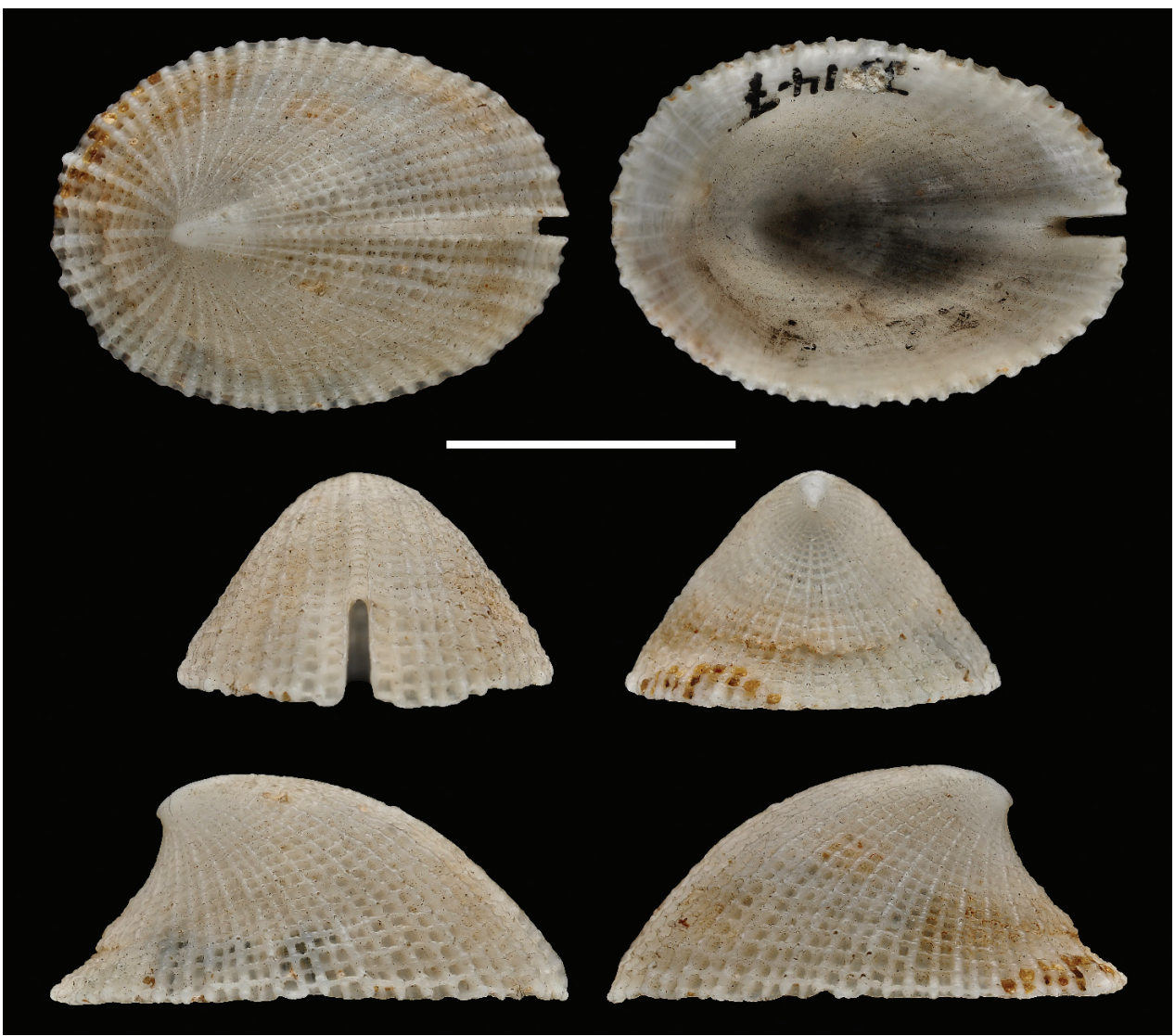

Figure 6. Emarginula koon Kilburn, 1978. Holotype T2196/NMSA-MOL 0B147. Scale bar: 10 mm. 
South Africa - KwaZulu-Natal, Port Shepstone; H. Burnup leg.; T2194; NMSA-MOL 003067.

South Africa - Eastern Cape, Mzamba; 25-26 Sep. 1976; R. Kilburn leg.; T2151; NMSA-MOL 0A6238.

South Africa - KwaZulu-Natal, Durban; J.F. Quekett, collection of Burnup; T2198; NMSA-MOL 001280.

South Africa • KwaZulu-Natal, south coast, Palm Beach; R. Cock leg.; T2197; NMSA-MOL 006385.

Current status. Emarginula koon Kilburn, 1978; original combination.

\section{Emarginula mcclurgi Kilburn, 1978}

macclurgi Kilburn, 1978: 443-444, Pl. 7 [Emarginula, off St Lucia Lighthouse, Zululand, in $100 \mathrm{~m}$, mud and pebble bottom (C.S.I.R Water Research bottom sample): $8.7 \times 6.7 \times 4.0 \mathrm{~mm}]$.

Material examined. Holotype. South Africa - KwaZulu-Natal, St Lucia Lighthouse, depth $100 \mathrm{~m}$, station F3; ex C.S.I.R Water Research; mud and pebbles; T2190; NMSA-MOL 0A5726; Fig. $7(8.5 \times 6.7 \times 4.1 \mathrm{~mm})$.

Current status. Emarginula macclurgi Kilburn, 1978; original combination.

Remarks. Subsequent to the original description, at which time Emarginula macclurgi was only known by a single specimen, this species has been collected along the eastern coast of South Africa, from Mission Rocks in the northern KwaZulu-Natal $\left(28^{\circ} 17.5^{\prime} \mathrm{S}, 32^{\circ} 34.2^{\prime} \mathrm{E}\right)$ to Mbashe River in the south $\left(32^{\circ} 20.6^{\prime} \mathrm{S}, 29^{\circ} 00.2^{\prime} \mathrm{E}\right)$ in depths ranging from 60 to $175 \mathrm{~m}$.

\section{Emarginula phrygium Herbert \& Kilburn, 1986}

phrygium Herbert \& Kilburn, 1986: 10, figs 30-34 [Emarginula, off Qora River, Transkei $\left(32^{\circ} 34.0^{\prime} \mathrm{S}, 28^{\circ} 49.7^{\prime} \mathrm{E}\right)$, depth $400-420 \mathrm{~m}$. Coarse, slightly muddy sand (dredged Meiring Naudè): 5.8×4.7, height $3.7 \mathrm{~mm}$ ].

Material examined. Holotype. South Africa • Eastern Cape, off Qora River, $32^{\circ} 34.0^{\prime}$ S, $28^{\circ} 49.7^{\prime} \mathrm{E}$, depth 400-420 m, station U13, 12 Jul. 1984; dredged MN; coarse slightly muddy sand; T3012; NMSA-MOL 0C0684; Fig. 8 (5.68×4.67×3.6 mm).

Current status. Emarginula phrygium Herbert \& Kilburn, 1986; original combination.

\section{Emarginula thorektes Kilburn, 1978}

thorektes Kilburn, 1978: 440-442, Pl. 6a,b (holotype), 6c (paratype) [Emarginula, Shelly Beach, near Port Shepstone (leg. L. and R. Cock): 8.7×5.8×4.0 mm]. 


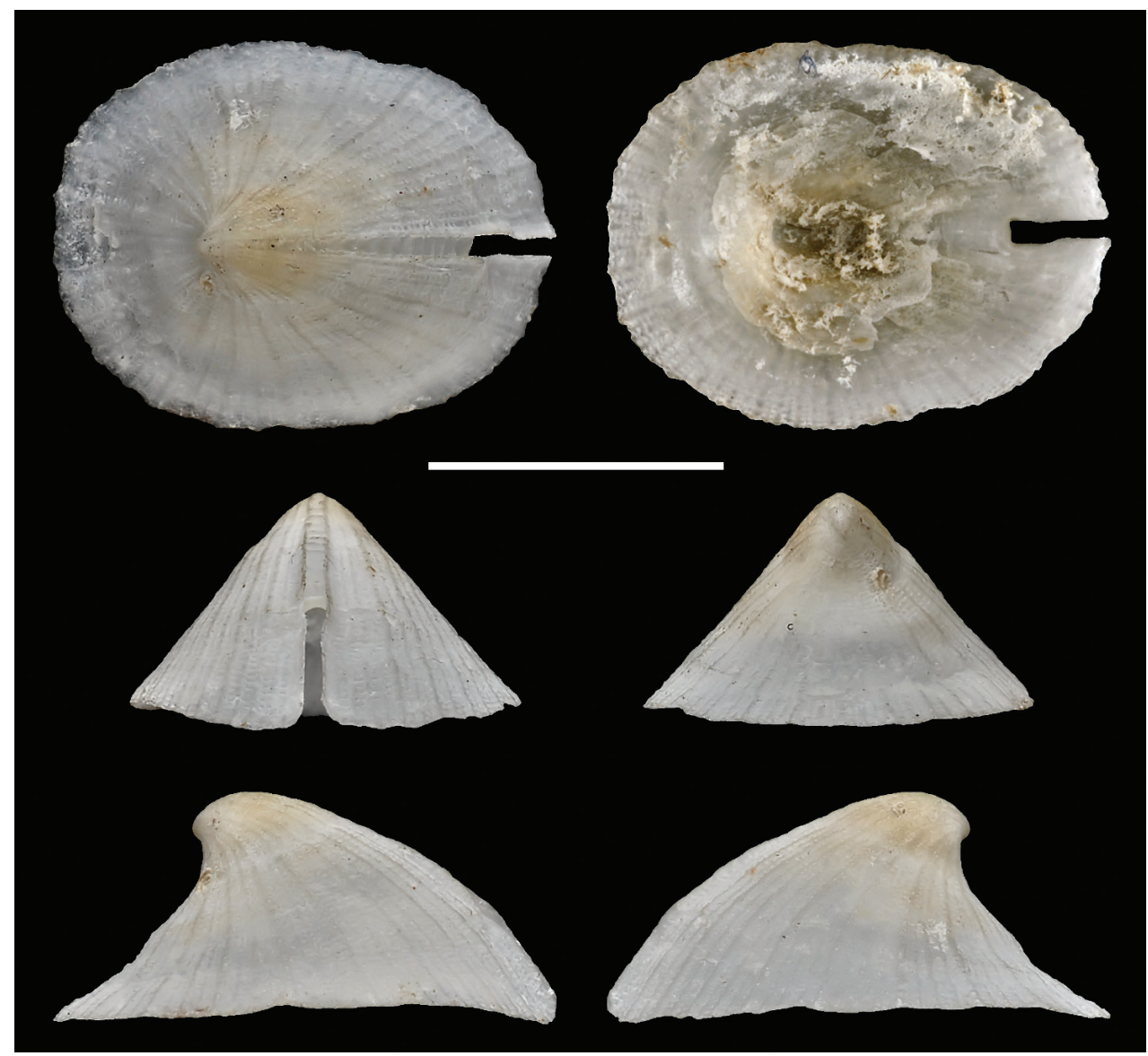

Figure 7. Emarginula mcclurgi Kilburn, 1978. Holotype T2190/NMSA-MOL 0A5726. Scale bar: 5 mm.

Material examined. Holotype. South Africa - KwaZulu-Natal, Shelly Beach, near Port Shepstone; don. Mrs. R. Cock, Nov. 1975; T2193; NMSA-MOL 0A3829; Fig. 9 $(8.5 \times 5.7 \times 3.4 \mathrm{~mm})$.

Paratypes. South Africa • juvenile; KwaZulu-Natal, off Sodwana Bay, depth 50 m, ex C.S.I.R. Department of Water Research; shell and coral sand, foraminifera; T2191; NMSA-MOL 0A5763.

South Africa - KwaZulu-Natal, off Port Edward, depth 100 m; 18 Oct. 1976; ex C.S.I.R. Department of Water Research; fine shell sand; T2192; NMSAMOL 0A5764.

Current status. Emarginula thorektes Kilburn, 1978; original combination.

Remarks. One paratype (T2192/NMSA-MOL 0A5764) is Emarginula connelli Kilburn, 1978 (first noticed by D. Herbert, as indicated in his handwriting in one of the reprints of the original descriptions of thorektes and connelli and on the additional label). 


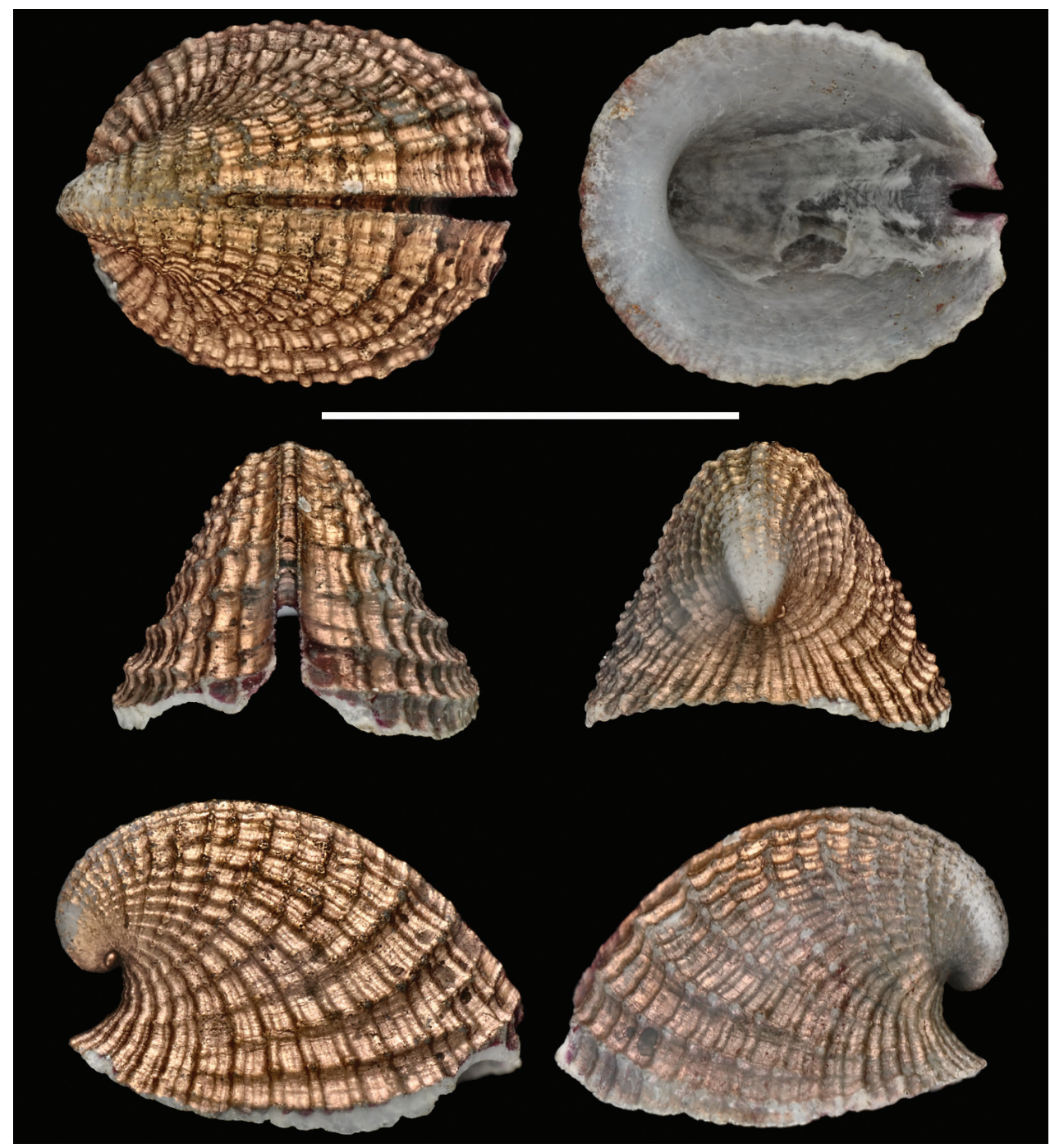

Figure 8. Emarginula phrygium Herbert \& Kilburn, 1986. Holotype T3012/NMSA-MOL 0C6844. Scale bar: $5 \mathrm{~mm}$.

\section{Emarginula viridicana Herbert \& Kilburn, 1986}

viridicana Herbert \& Kilburn, 1986: 12, figs 45, 46 (holotype), 47, 49-50 (paratypes) [Emarginula, off Park Rynie (approx. 30 $21^{\prime} \mathrm{S}, 30^{\circ} 51^{\prime} \mathrm{E}$ ), living, $110 \mathrm{~m}$, sponge rubble. Dredged MN: 15.1×10.6, height $9.4 \mathrm{~mm}$ ].

Material examined. Holotype. South AfriCa • soft parts in ethanol; KwaZulu-Natal, off Park Rynie, depth 110 m, dredged; 2 Mar. 1981; R. Kilburn leg.; sponge rubble; T3021; NMSA-MOL 0B8749; Fig. $10(15.2 \times 10.53 \times 9.3 \mathrm{~mm})$. 


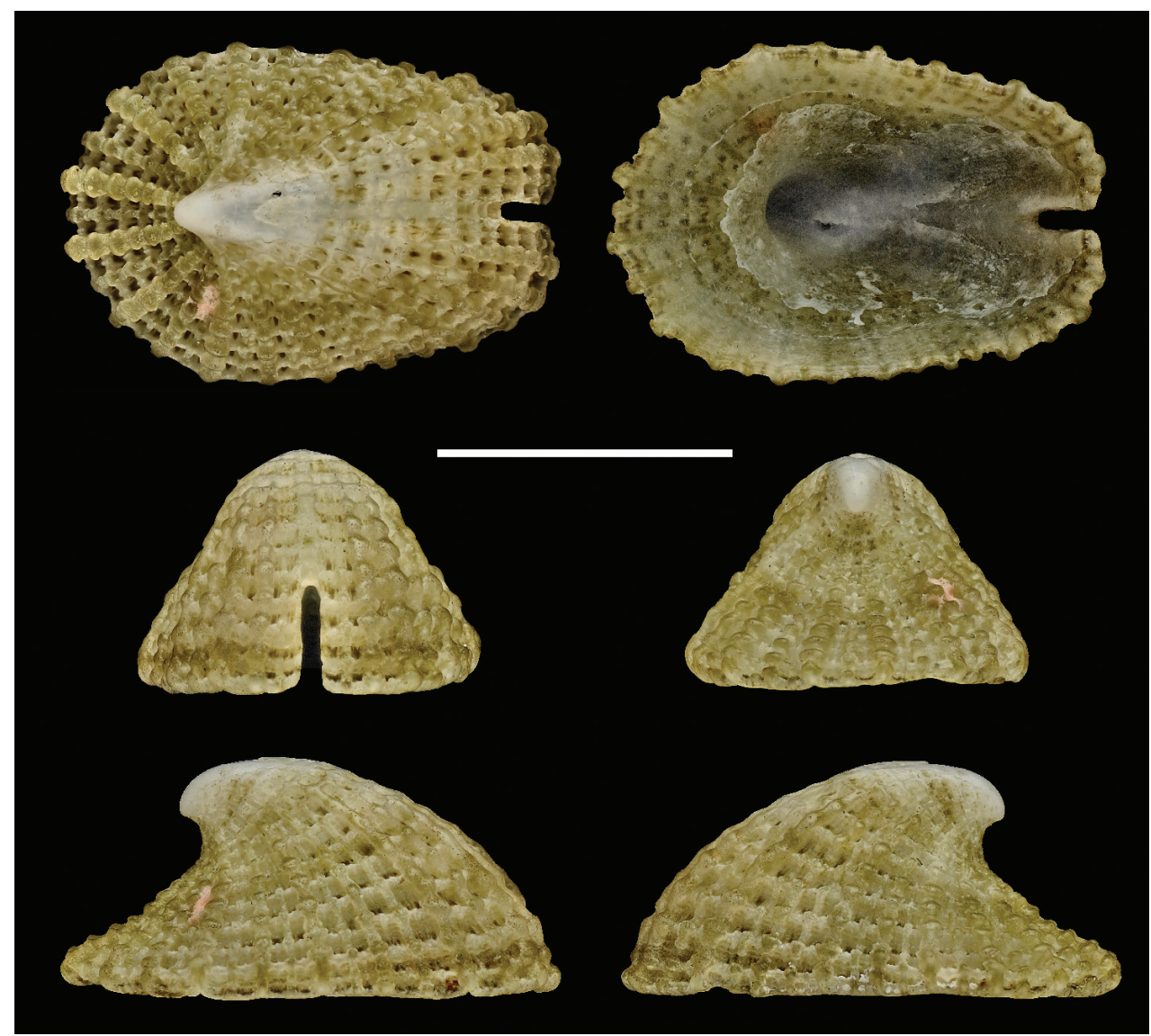

Figure 9. Emarginula thorektes Kilburn, 1978. Holotype T2193/NMSA-MOL 0A3829. Scale bar: 5 mm.

Paratypes. South Africa • soft parts in ethanol; same data as holotype; T3022; NMSA-MOL 0B9937.

South Africa • 2; dried soft parts; KwaZulu-Natal, off Park Rynie, depth 100 m; 2 Mar. 1981; dredged, R. Kilburn; sand and sponge rubble; T3023; NMSA-MOL 0B8750.

South Africa • dried soft parts; KwaZulu-Natal, off Park Rynie, depth 120 m, 2 Mar. 1981; dredged, R. Kilburn; rubble and solitary coral; T3024; NMSAMOL 0B3800.

South Africa - KwaZulu-Natal, off Park Rynie, 30²2.8'S, 3051.3'E, depth 142 m, station X5; 19 Aug. 1981; dredged MN; some sand, sponge rubble; T3025; NMSA-MOL 0B8745; figured (fig. 47) in the original description.

South Africa • KwaZulu-Natal, offTrafalgar, 31 $01.2^{\prime}$ S, 30²2.9'E, depth $120 \mathrm{~m}$, station X4; 22 Jul. 1982; dredged MN; sand, sponge; T3026; NMSA-MOL 0B8762.

South Africa • soft parts in ethanol; KwaZulu-Natal, off Park Rynie, 30²3.8'S, 3050.0'E, depth $101 \mathrm{~m}$, station X10; 19 Aug. 1981; dredged MN; some sand, sponge rubble; T3027; NMSA-MOL 0C1557. 


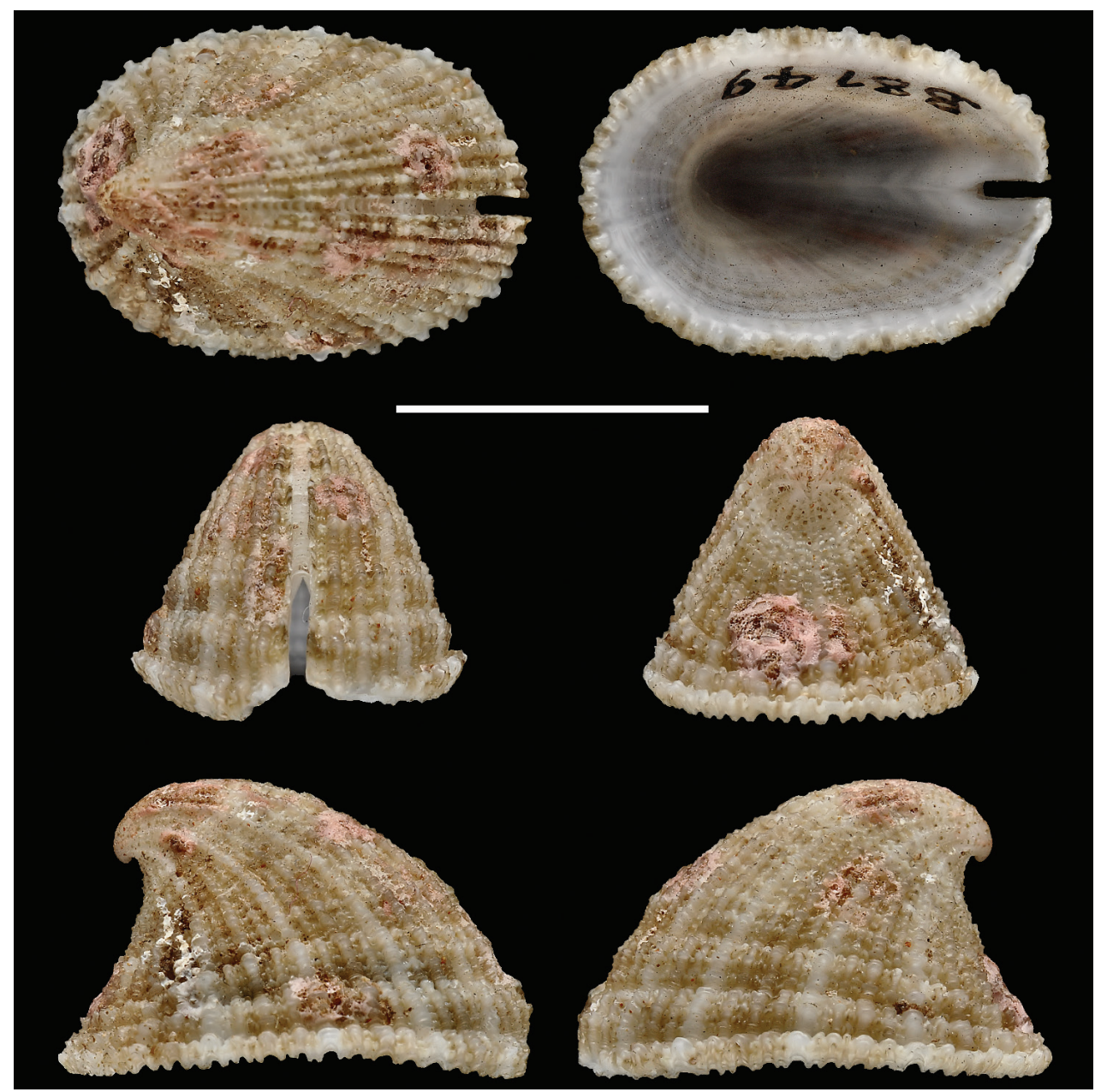

Figure 10. Emarginula viridicana Herbert \& Kilburn, 1986. Holotype T3021/NMSA-MOL 0B8749. Scale bar: $10 \mathrm{~mm}$.

South Africa - KwaZulu-Natal, off Park Rynie, 30²0.7'S, 3051.6'E, depth 105 m, station XX18; 10 Jul. 1984; dredged MN; sponge rubble; T3028; NMSAMOL 0B8541; figured (fig. 49) in the original description.

South Africa - soft parts in ethanol; KwaZulu-Natal, off Park Rynie, depth 100 m; 5 Mar. 1981; dredged, R. Kilburn sand; T3029; NMSA-MOL 0B8746; figured (fig. 50) in the original description.

South Africa • KwaZulu-Natal, off Park Rynie, depth 100 m; 4 Mar. 1981; dredged, R. Kilburn; sand, sponge rubble; T3036; NMSA-MOL 0B3726.

South Africa • Eastern Cape, off Mzamba River, 31 $1^{\circ} 06.0^{\prime} \mathrm{S}, 30^{\circ} 18.3^{\prime} \mathrm{E}$, depth 100 m, station XX2; 15 Jun. 1983; dredged MN; sponge rubble; T3035; NMSAMOL 0C7102. 
Currentstatus. Emarginula viridicana Herbert \& Kilburn, 1978; original combination.

Remarks. One paratype (NMSA-MOL 0B3800), of two, has been sent to NHMUK (registration number: NHMUK 1986090). Some specimens, including the holotype, were collected in March 1981 during one of the trial runs on Meiring Naudé prior to the first official Meiring Naudé research cruise in August 1981 (David G. Herbert, personal communication).

\section{Puncturella (Vacerrena) christiaensi Kilburn, 1978}

christiaensi Kilburn, 1978: 448, Plate 9c-e, 10 [Puncturella (Vacerrena), off Sordwana [sic] Bay, Zululand, $50 \mathrm{~m}$, in shell grit (C.S.I.R. Department of Water Research): $-1.9 \times \mathrm{n} / \mathrm{d} \times 1.5 \mathrm{~mm}$, basal length $1.65 \mathrm{~mm}$ ].

Material examined. Holotype. South Africa - KwaZulu-Natal, off Sodwana Bay, depth 50 m, C.S.I.R. Water Res; shell grit; T2204; NMSA-MOL 0B230; Fig. 11 $(1.92 \times 1.45 \times 1.41 \mathrm{~mm}$; larger diameter (height) of aperture ("basal length") is $1.52 \mathrm{~mm})$.

Paratypes. South Africa -3 of 5; same data as holotype; T2205; NMSAMOL 0A5084.

South Africa • 2; KwaZulu-Natal, off Kosi Bay, depth 50 m; C.S.I.R. Water Res; in shell drift; T2206; NMSA-MOL 0A5937.

Current status. Vacerrena nana (H. Adams, 1872); Herbert (2015).

Remarks. The shell of the holotype was damaged after the photos for the original description were made. The damage was first discovered in March 2012 by the first author and the part that was broken off was not located. Another set of dimensions $(1.6 \times 1.5 \mathrm{~mm})$ is given in the figure captions for the plate $9(\mathrm{c}-\mathrm{e})$ in the original description. We were unable to locate two out of five paratypes (T2205/ NMSA 0A5084). Genus Vacerrena is probably related to Cornisepta (Fig. 12) and Profundisepta (Fig. 13) but is not transferred here to Zeidorinae because it is not a taxonomic revision and there is no reference that can be cited to indicate that it should be in Zeidorinae.

\section{Subfamily Zeidorinae Naef, 1913}

\section{Fissurisepta onychoides Herbert \& Kilburn, 1986}

onychoides Herbert \& Kilburn, 1986: 24, figs 87 (holotype), 88-89 (paratype) [Fissurisepta, off Shixini Point, Transkei, $32^{\circ} 31.2^{\prime} \mathrm{S}, 28^{\circ} 52.2^{\prime} \mathrm{E}, 300 \mathrm{~m}$, course sand, broken shell. Dredged MN: 3.6×2.6 mm, height 4.9].

Material examined. Holotype. South Africa - Eastern Cape, off Shixini point, $32^{\circ} 31.2^{\prime} \mathrm{S}$, 28 $8^{\circ} 52.2^{\prime} \mathrm{E}$, depth $300 \mathrm{~m}$, station T14; 11 Jul. 1984; dredged MN; broken shell, course sand; T3017; NMSA-MOL 0C6365; Fig. $12(3.65 \times 2.59 \times 5.01 \mathrm{~mm})$. 


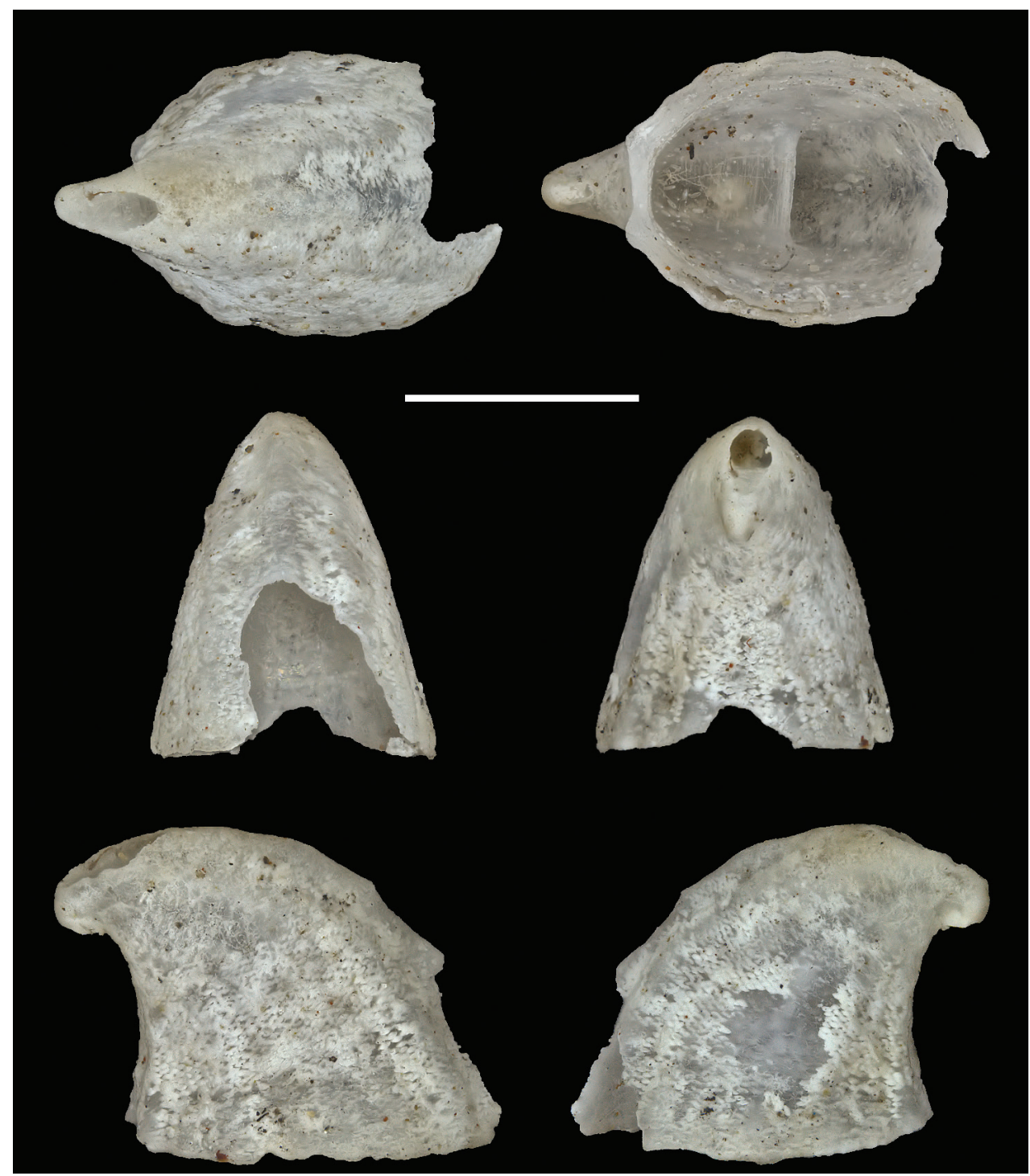

Figure II. Puncturella (Vacerrena) christiaensi Kilburn, 1978. Holotype T2204/NMSA-MOL 0B230. Scale bar: $1 \mathrm{~mm}$.

Paratypes. South Africa • Eastern Cape, off Rame Head, 31 ${ }^{\circ} 57.3^{\prime}$ S, 292 25.5'E, depth 380 m, station K14; 20 Jul. 1982; dredged MN; coarse sand, old shell debris; T3016; NMSA-MOL 0C7156; figured (figs 88, 89) in the original description.

South Africa - same data as NMSA-MOL 0C7156; T3015; NMSAMOL 0C2092.

South Africa • Eastern Cape, off Rame Head, 31 $56.1^{\prime}$ 'S, $29^{\circ} 26.5^{\prime}$ E, depth 410430 m, station K13; 20 Jul. 1982; dredged MN; stones, some sand; T3031; NMSAMOL 0C7201. 


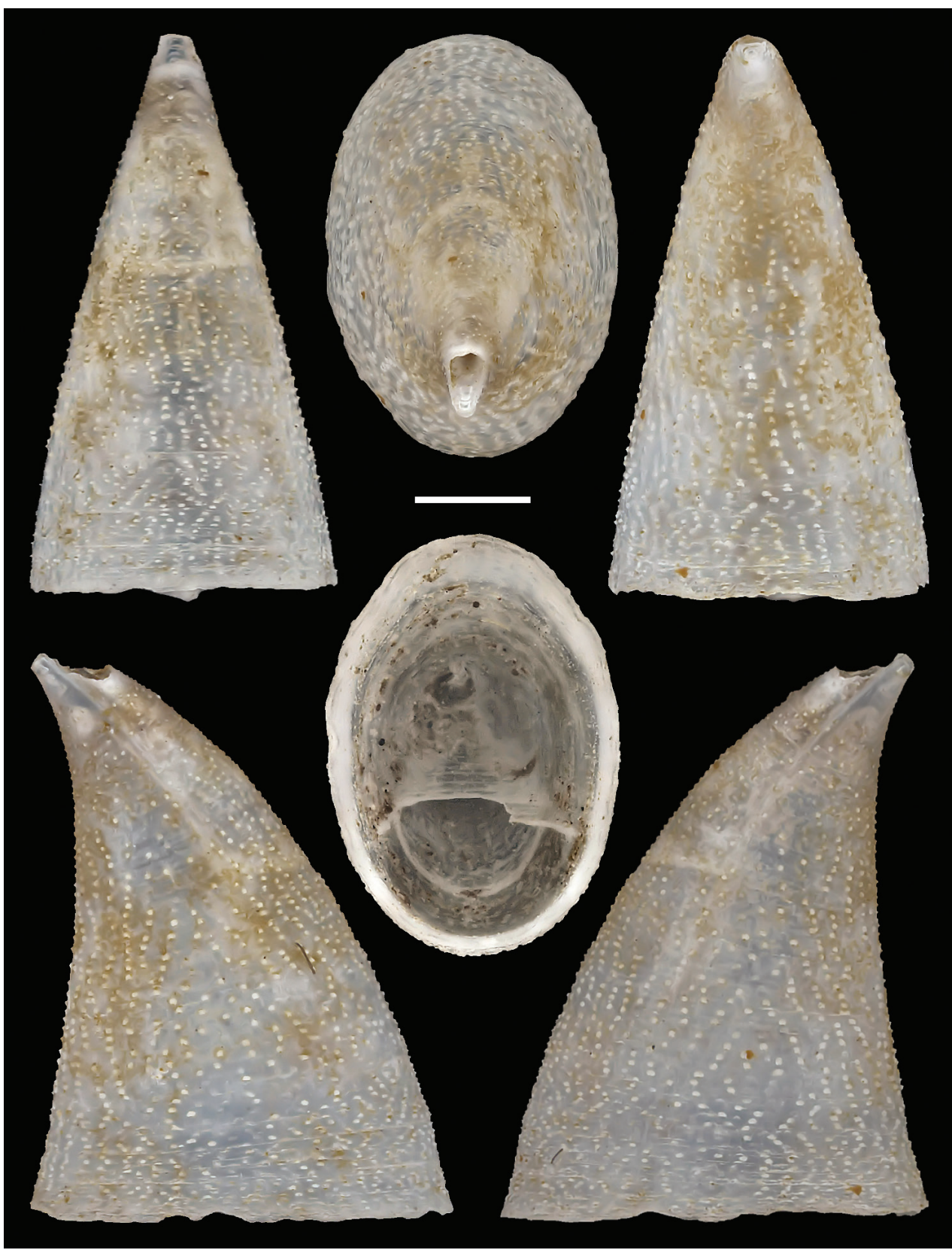

Figure I2. Fissurisepta onychoides Herbert \& Kilburn, 1986. Holotype T3017/NMSA-MOL 0C6365. Scale bar: $1 \mathrm{~mm}$.

South Africa • Eastern Cape, off Whale Rock, 3200.9'S, 29²1.8'E, depth 400420 m, station M7, 20 Jul. 1982; dredged MN; coarse sand, old shell debris, stones; T3033; NMSA-MOL 0C7161. 
South Africa • 3; Eastern Cape, off Stony Point, 32³7.5'S, 2845.8'E, depth 390-400 m, station V11; 12 Jul. 1984; dredged MN; muddy sand, small stones; T3034; NMSA-MOL 0C7175.

South Africa - Eastern Cape, off Mgazi River, 3144.8'S, 29³3.0'E, depth 370 m, station J8; 4 Jul. 1985; dredged MN; soft black mud, few rocks, large crinoids; T3086; NMSA-MOL 0C8823.

South Africa •3 Eastern Cape, off Mbashe River, 32²3.6'S, 2859.2'E, depth 295-350 m, station Q17; 6 Jul. 1985; dredged MN; coarse sand; T3087; NMSAMOL 0C9096.

South Africa • 5 of 6; KwaZulu-Natal, off Umlaas Canal, 3002.2'S, 31 ${ }^{\circ} 03.9^{\prime} \mathrm{E}$, depth 250 m, station XX67; 9 Jul. 1985; dredged MN; coarse sand; T3085; NMSAMOL 0D1421.

South Africa - 4 of 8 or 7; KwaZulu-Natal, off Amanzimtoti, 3004.7'S, $31^{\circ} 03.3^{\prime} \mathrm{E}$, depth 300-305 m, station XX66; 9 Jul. 1985; dredged MN; medium sand; T3084; NMSA-MOL 0D1312.

Current status. Cornisepta onychoides (Herbert \& Kilburn, 1986); McLean and Geiger (1998); Herbert (2015).

Remarks. One paratype (T3085/NMSA-MOL 0D1421) was sent to Zoological Museum of Moscow University in 1990. Two, of supposedly eight, paratypes (T3084/ NMSA-MOL 0D1312) have been sent to NHMUK (registration number: NHMUK 1986088) and one from the same lot, - to Humboldt University, Berlin (however, probably due to initial miscount, only four, not five, remain in NMSA).

\section{Puncturella (Puncturella) voraginosa Herbert \& Kilburn, 1986}

voraginosa Herbert \& Kilburn, 1986: 18, figs 66-70 [Puncturella (Puncturella s.1.), off Port Grosvenor, Transkei (29 57.6'S, 31 $\left.26.2^{\prime} \mathrm{E}\right)$ [sic], 100-115 m, sand, some mud, solitary corals and shells. Dredged MN: $2.3 \times 1.7 \mathrm{~mm}$, height $1.3 \mathrm{~mm}$ ].

Material examined. Holotype. South Africa - Eastern Cape, off Port Grosvenor, $31^{\circ} 26.2^{\prime} \mathrm{S}, 29^{\circ} 57.6^{\prime} \mathrm{E}$, depth 100-115 m, station D3; Aug. 1981; dredged MN; sand, some mud, solitary corals, shells; T3020; NMSA-MOL 0C1296; Fig. 13 $(2.38 \times 1.82 \times 1.32 \mathrm{~mm})$.

Paratypes. South Africa • Eastern Cape, off Rame Head, 31 $56.1^{\prime}$ S, 2926.5'E, depth 410-430 m, station K13; 20 Jul. 1982; dredged MN; stones, some sand; T3019; NMSA-MOL 0C7045.

South Africa • same data as paratype NMSA-MOL 0C7045; T3032; NMSAMOL 0C7203.

South Africa • same data as paratype NMSA-MOL 0C7045; T3089; NMSAMOL 0C7998.

South Africa • Eastern Cape, off Mgazi River, depth 180 m, 31ํ⒋7'S, 29³3.7'E, station J1; 4 Jul. 1985; dredged MN; soft mud; T3040; NMSA-MOL 0C7355. 


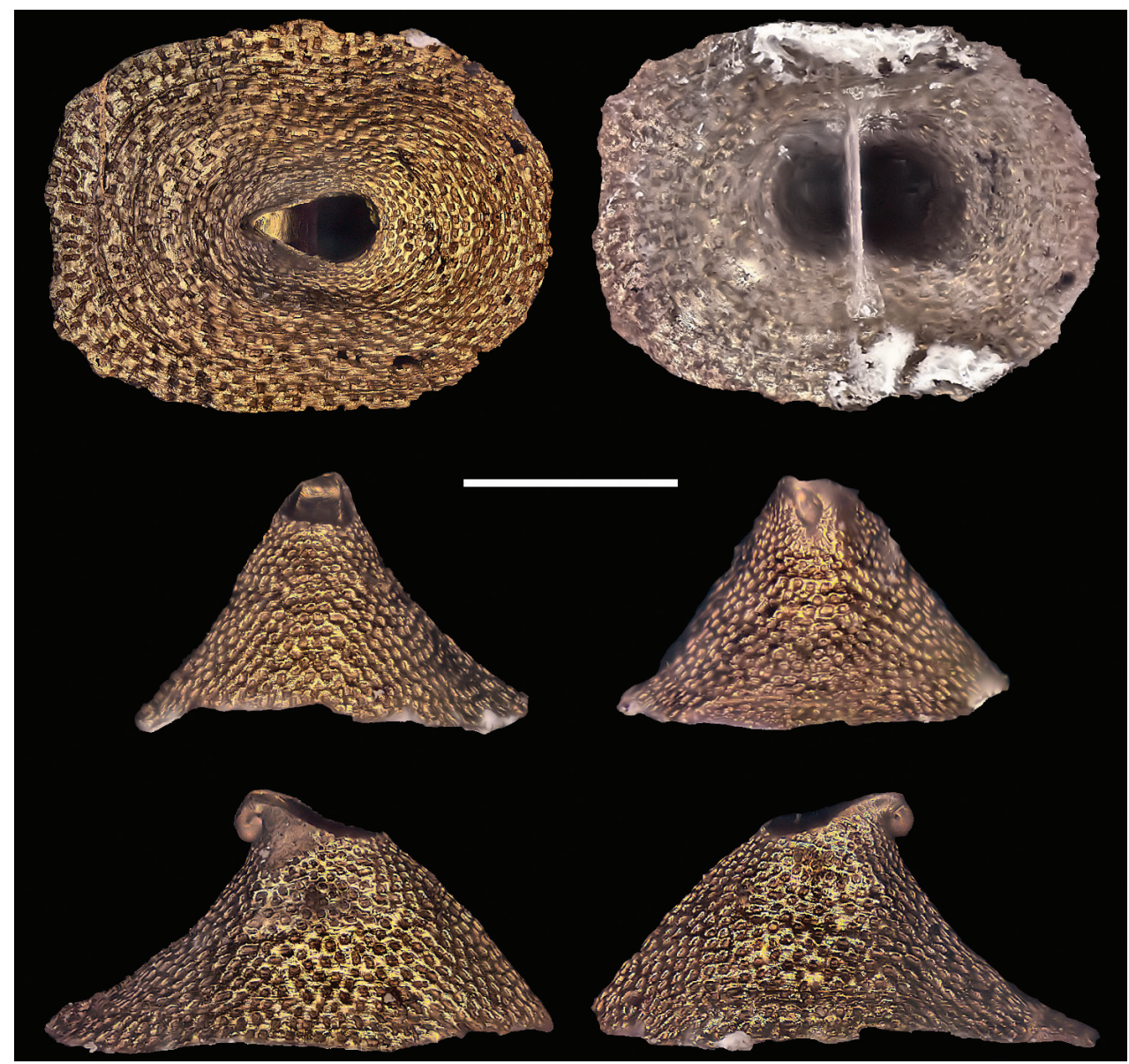

Figure 13. Puncturella (Puncturella) voraginosa Herbert \& Kilburn, 1986. Holotype T3020/NMSAMOL 0C1296. Scale bar: $1 \mathrm{~mm}$.

South Africa - Eastern Cape, off Mgazi River, 3144.8'S, 29³3.0'E, depth 370 m, station J8, 4 Jul. 1985; dredged MN; soft black mud, few rocks, large crinoids; TI083; NMSA-MOL 0C8824.

Current status. Profundisepta voraginosa (Herbert \& Kilburn, 1986); Herbert (2015).

Remarks. South and East were reversed for the coordinates in the original description (that would place type locality to incorrect position $\sim 40 \mathrm{~km}$ ESE off Durban at $438 \mathrm{~m}$ depth).

\section{Puncturella (Granopsis) serraticosta Herbert \& Kilburn, 1986}

serraticosta Herbert \& Kilburn, 1986: 20, figs 71-74 [Puncturella (Cranopsis), off Shixini Point, Transkei (32³1.6'S, 28 $\left.2^{\circ} 53.0^{\prime} \mathrm{E}\right), 500 \mathrm{~m}$. Dredged MN: 10,0×6,9 mm, height $6,4 \mathrm{~mm}$ ]. 
Material examined. Holotype. South Africa - Eastern Cape, off Shixini point, $32^{\circ} 31.6^{\prime} \mathrm{S}, 28^{\circ} 53.0^{\prime} \mathrm{E}$, depth $500 \mathrm{~m}$; station T17; 13 Jul. 1984; dredged MN; muddy sand, coral rubble; T3013; NMSA-MOL 0C7064; Fig. $14(10 \times 6.9 \times 6.5$ mm).

Paratypes. South Africa • same data as holotype; T3014; NMSA-MOL 0C7155.

Current status. Puncturella serraticosta Herbert \& Kilburn, 1986; original combination.

Remarks. Another set of dimensions of the holotype is indicated in the figure caption of the original description: " $9.9 \times 6.9 \mathrm{~mm}$, height $6.3 \mathrm{~mm}$ ". Cranopsis A. Adams, 1860 has been synonymised with Puncturella R. T. Lowe, 1827 (Cunha et al. 2019).

\section{Subfamily Rimulinae Anton, 1838}

\section{Rimula rhips Herbert \& Kilburn, 1986}

rhips Herbert \& Kilburn, 1986: 25, figs 84-86 [Rimula, off Port Grosvenor, Transkei $\left(39^{\circ} 57.2^{\prime} \mathrm{S}, 31^{\circ} 25.9^{\prime} \mathrm{E}\right)$ [sic], 120-128 m, coarse sand, some mud, solitary coral, shells. Dredged MN: $9.4 \times 6.7 \times$ height $4.1 \mathrm{~mm}]$.

Material examined. Holotype. South Africa - Eastern Cape, Port Grosvenor, 31 $25.9^{\prime}$ S, 29 57.9'E, depth 120-128 m, station D2; Aug. 1981; dredged MN; coarse sand, some mud, solitary coral, shells; T3018; NMSA-MOL 0C1192; Fig. 15 $(9.33 \times 6.67 \times 3.87 \mathrm{~mm})$.

Current status. Rimula rhips Herbert \& Kilburn, 1986; original combination.

Remarks. The coordinates in the original description were incorrectly recorded; latitude and longitude were swopped, and it should be $29^{\circ} \mathrm{E}$ instead of $39^{\circ} \mathrm{S}$. Subsequent to the original description, at which time Rimula rhips was only known by a single specimen, this species has been collected between 40 and $128 \mathrm{~m}$ deep up to Boteler Point, $27^{\circ} 01.1^{\prime} \mathrm{S}, 32^{\circ} 55.2^{\prime} \mathrm{E}$, close to the border between South Africa and Mozambique.

\section{Subfamily Fissurellinae J. Fleming, 1822}

\section{Amblychilepas platyactis McLean \& Kilburn, 1986}

platyactis McLean \& Kilburn, 1986: 9-11, figs 3 (paratype), 24 (holotype), 25 (paratype NMSA 6769/T3009) [Amblychilepas, Port Alfred, Eastern Cape Province, South Africa, collected by R. Kilburn,1966: $17.2 \times 10.3 \times 3.9 \mathrm{~mm}]$.

Material examined. Holotype. South Africa • soft parts in ethanol; Eastern Cape, Port Alfred; collected by R. Kilburn, 1966, donated in Sep. 1969; T2744; NMSAMOL 0B6397; Fig. $16(16.8 \times 10.13 \times 4$ mm).

Paratypes. South Africa • soft parts in ethanol; Western Cape: Cape Peninsula, Kommetjie, C.M. Connolly leg.; T3008; NMSA-MOL 0B9916. 


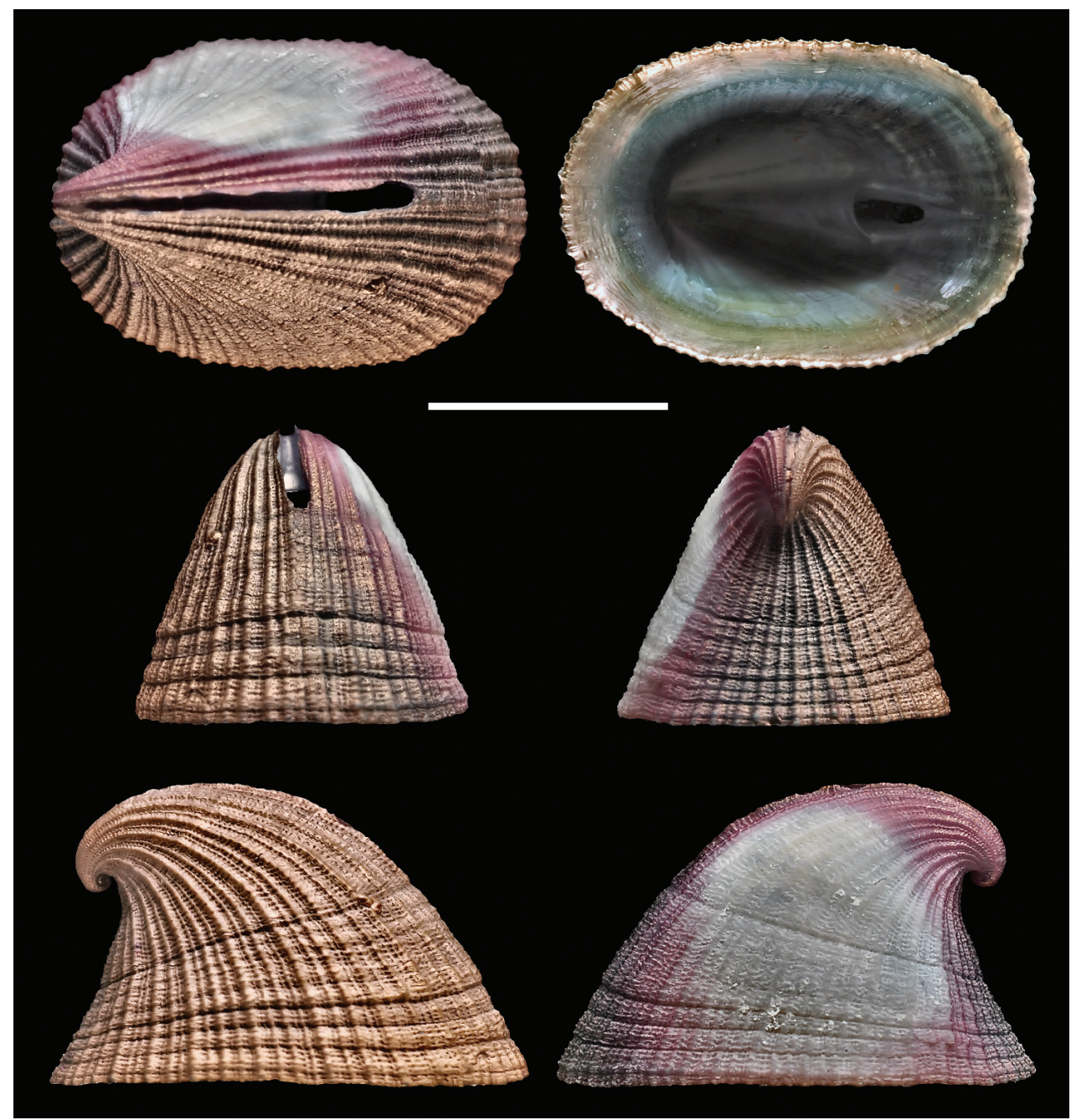

Figure 14. Puncturella (Granopsis) serraticosta Herbert \& Kilburn, 1986. Holotype T3013/NMSA-MOL 0C7064. Scale bar: $5 \mathrm{~mm}$.

South Africa - 1 of 3, soft parts in ethanol; Eastern Cape, E. of East London, Kwelera; C.M. Connolly leg.; T3058; NMSA-MOL 0B9929; SEM of radula (largest); alc. vial also contains radula pieces; figured (fig. 3) in the original description.

South Africa • 6; Western Cape, False Bay, Simonstown, C.M. Connolly leg.; T3009; NMSA-MOL 006769; figured (fig. 25) in the original description.

Current status. Amblychilepas platyactis McLean \& Kilburn, 1986; original combination.

Remarks. It could be difficult to recognise anterior and posterior ends in a side view of a shell of this species. The original description states that shell is "slightly narrowed anteriorly" (McLean and Kilburn 1986: 9) that makes it flatter (not higher) posteriorly. Only one of three of paratypes T3058/NMSA-MOL 0B9929 is in NMSA. 


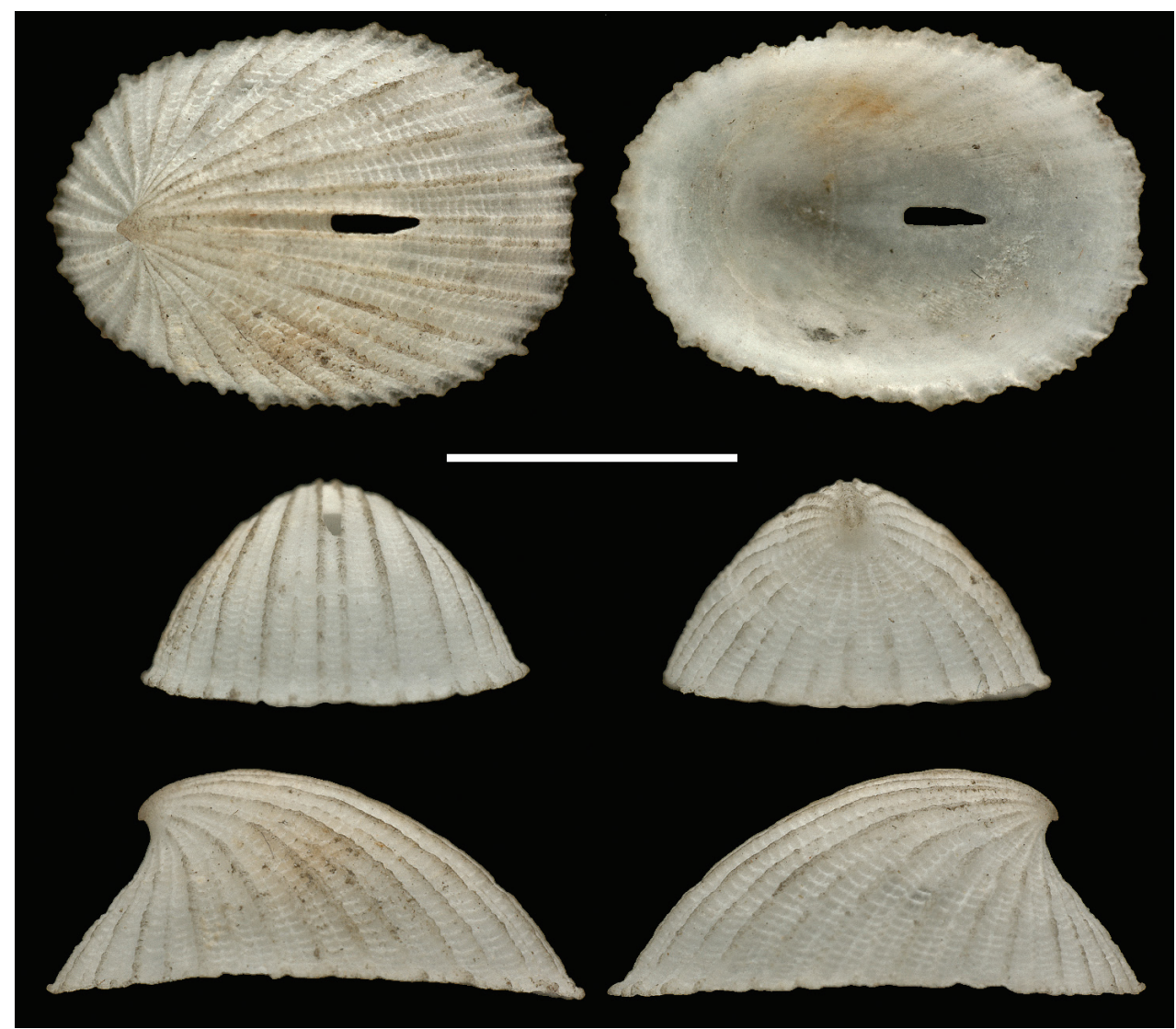

Figure 15. Rimula rhips Herbert \& Kilburn, 1986. Holotype T3018/NMSA-MOL 0C1192. Scale bar: $5 \mathrm{~mm}$.

\section{Superfamily Scissurelloidea Gray, 1847} Family Anatomidae McLean, 1989

\section{Scissurella agulhasensis Thiele, 1925}

agulhasensis Thiele, 1925: 41(7), Pl. 13(I), fig. 2 [Scissurella, From station $104\left(35^{\circ} 16^{\prime}\right.$ S, $22^{\circ} 26.7^{\prime} \mathrm{E}, 155 \mathrm{~m}$, in the Agulhas current (S.S. Valdivia): diameter $2.5 \mathrm{~mm}$ ].

Material examined. Neotype. South Africa - Eastern Cape, off Mtamvuna River, $31^{\circ} 09.7^{\prime} \mathrm{S}, 30^{\circ} 15.3^{\prime} \mathrm{E}$, depth $120-140 \mathrm{~m}$, station A7; Aug. 1981; dredged MN; sponge rubble; T1916; NMSA-MOL 0W181 (ex NMSA C7967); Fig. $17(2.28 \times 2.1 \times 2.1 \mathrm{~mm})$.

Current status. Anatoma agulhasensis (Thiele, 1925); Geiger (2012).

Remarks. Neotype designated by Geiger and Jansen (2004: 6) because three syntypes hosted in the Zoological Museum Berlin were destroyed through Bynesian decay. Neotype was separated from NMSA C7967 (figured by Herbert 1986: 611-613, figs 3, 12, 14). Re-described by Geiger in 2012. 


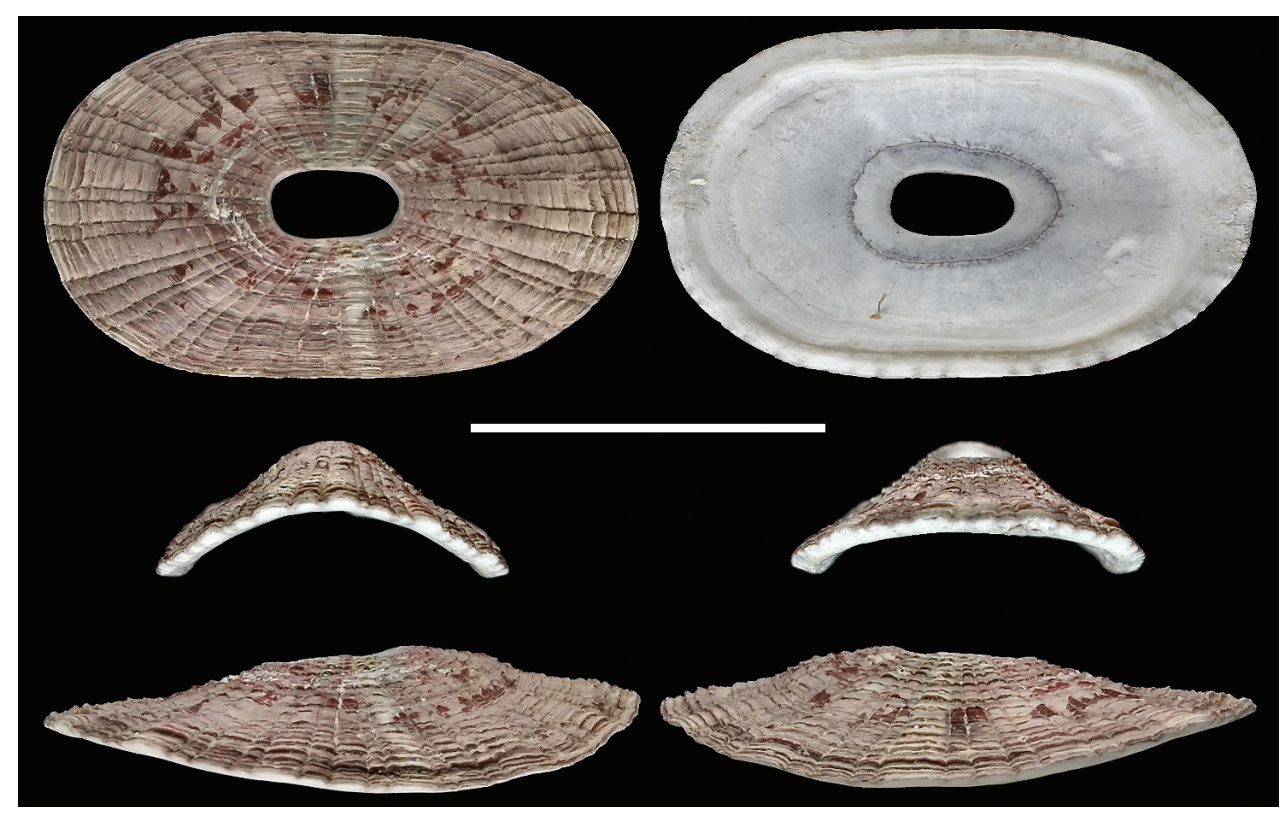

Figure I 6. Amblychilepas platyactis McLean \& Kilburn, 1986. Holotype T2744/NMSA-MOL 0B6397. Scale bar: $10 \mathrm{~mm}$.

\section{Anatoma yaroni Herbert, 1986}

yaroni Herbert, 1986: 610, 613-617, figs 1, 2 (radula), 4 (paratype), 15,17 (paratype), 16 (holotype) [Anatoma, off Shixini Point, Transkei (32 $\left.31.7^{\prime} \mathrm{S}, 28^{\circ} 52.7^{\prime} \mathrm{E}\right), 490$ m, muddy sand, coral rubble (dredged Meiring Naude): $4.5 \times \mathrm{n} / \mathrm{d} \times 3.8 \mathrm{~mm}]$.

Material examined. Holotype. South Africa - Eastern Cape, off Shixini point, $32^{\circ} 31.7^{\prime} \mathrm{S}$, 28 $8^{\circ} 52.7^{\prime} \mathrm{E}$, depth $490 \mathrm{~m}$, station T18, 13 Jul. 1984; dredged MN; muddy sand, coral rubble; T3258; NMSA-MOL 0C6590; Fig. $18(4.46 \times 3.85 \times 4 \mathrm{~mm})$.

Paratypes. South Africa • same data as holotype; T3259; NMSA-MOL 0C9515.

South Africa • Eastern Cape, off Qora River, 32³5.4'S, 2849.2'E, depth 450-460 m, station U15; 14 Jul. 1984; dredged MN; sandy mud; T3260; NMSA-MOL 0C6641.

South Africa • Eastern Cape, off Whale Rock, 3202.0'S, 29²1.8'E, depth 430450 m, station M19; 3 Jul. 1985; dredged MN; fine muddy sand, T3261; NMSAMOL 0C9514.

South Africa • Eastern Cape, off Whale Rock, 3200.9'S, 29²1.8'E, depth 400420 m, station M7; 20 Jul. 1982; dredged MN; coarse sand, old shell debris, stones; T3262; NMSA-MOL 0C2046.

South Africa - 3; Eastern Cape, off Rame Head, 31 ${ }^{\circ} 57.3^{\prime}$ S, $29^{\circ} 25.5^{\prime} \mathrm{E}$, depth 380 m, station K14; 20 Jul. 1982; dredged MN; coarse sand, old shell debris; T3263; NMSA-MOL 0C9512. 


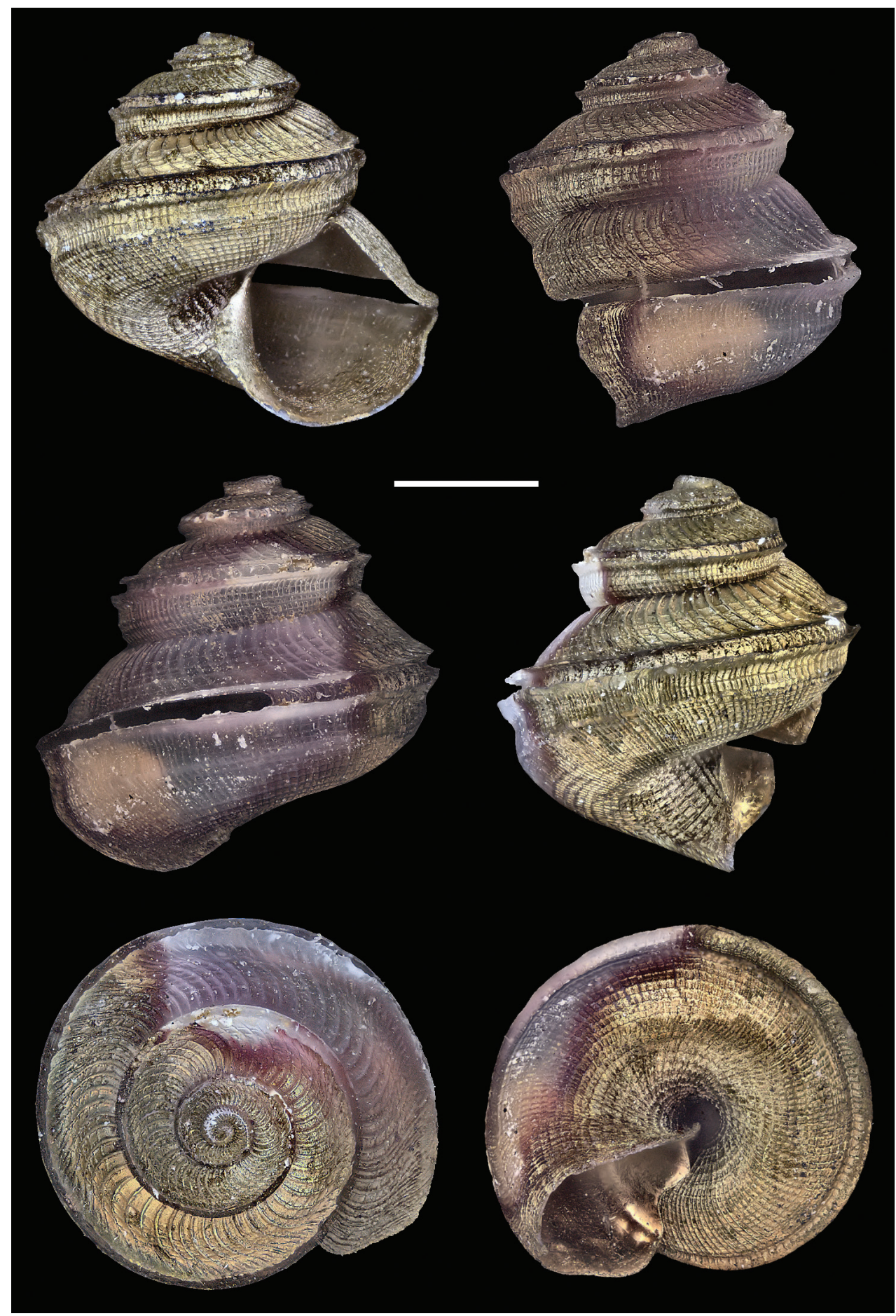

Figure 17. Scissurella agulhasensis Thiele, 1925. Neotype T1916/NMSA-MOL 0W181. Scale bar: $1 \mathrm{~mm}$. 


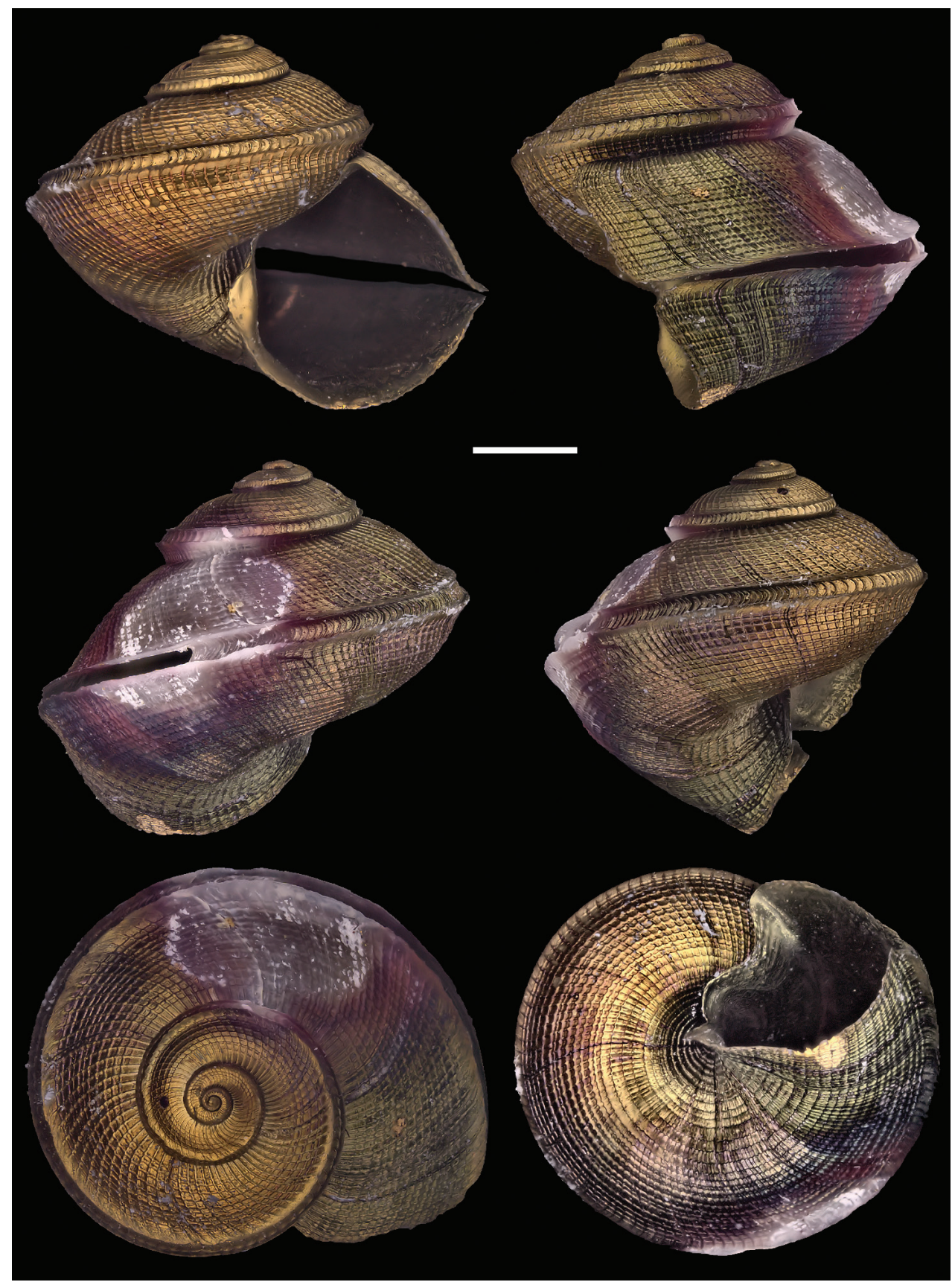

Figure 18. Anatoma yaroni Herbert, 1986. Holotype T3258/NMSA-MOL 0C6590. Scale bar: 1 mm.

South Africa • 3; Eastern Cape, off Stony Point, 32³7.8'S, 2845.9'E, depth 395 m, station V7; 14 Jun. 1983; dredged MN; sponge and stone; T3264; NMSAMOL 0C9511. 
South Africa - 2; Eastern Cape, off Kei River, 3250.0'S, 28³3.0'E, depth $450 \mathrm{~m}$, station Z7; 14 Jul. 1984; dredged MN; muddy sand with stones; T3265; NMSA-MOL 0C6904; figured (figs 15, 17) in the original description.

South Africa • 2; Eastern Cape, off Qolora River, 3247.6'S, 28³6.6'E, depth 510 m, station W13; 14 Jul. 1984; dredged MN; sandy mud; T3266; NMSAMOL 0C7010.

South Africa • Eastern Cape, off Mendu Point, 32²2.0'S, 2901.2'E, depth 405420 m, station R8; 8 Jun. 1983; dredged MN; fine sand; T3267; NMSA-MOL 0C4991.

South Africa - Eastern Cape, off Mbashe River, 32²3.6'S, 2859.2'E, depth 295-350 m, station Q17; 6 Jul. 1985; dredged MN; coarse sand; T3268; NMSAMOL 0C9513.

South Africa - 2; Eastern Cape, off Nthlonyane River, 32²17.4'S, 2905.6'E, depth 340-450 m, station P12; 5 Jul. 1985; dredged MN; dead Dendrophyllia; T3269; NMSA-MOL 0C8604.

South Africa - Eastern Cape, off Whale Rock, 3202.3'S, 29²19.9'E, depth 350 m, station M15; 3 Jul. 1985; dredged MN; fine muddy sand; T3270; NMSAMOL 0C9414; figured (fig. 4) in the original description.

South Africa • 12; Eastern Cape, off Shixini Point, 32³1.6'S, 2853.0'E, depth 500 m, station T17; 13 Jul. 1984; dredged MN; muddy sand, coral rubble; T3256 NMSA-MOL 0C9534.

South Africa • 8; same data as holotype; T3257; NMSA-MOL 0C9535.

Current status. Anatoma yaroni Herbert, 1986; original combination.

Remarks. Radula, illustrated on the figures 1, 2 in the original publication, is from the specimen NMSA-MOL 0C9007 listed in the "Additional material" on the page 615 of the original publication (David G. Herbert, personal communication).

\section{Family Scissurellidae Gray, 1847}

\section{Scissurella maraisorum Geiger, 2006}

maraisorum Geiger, 2006: 10-11, figs 7 (holotype), 8 (paratypes) [Scissurella, $20 \mathrm{~m}$, Aliwal Shoal, KwaZulu-Natal south coast, Republic of South Africa. June 2003. $30.250^{\circ} \mathrm{S}, 30.817^{\circ} \mathrm{E}$ (col. J.P. and A.P. Marais): $0.72 \mathrm{~mm}$ ].

Material examined. Holotype. South Africa - KwaZulu-Natal, Aliwal Shoal, $30.2507^{\circ} \mathrm{S}$, $30.8177^{\circ} \mathrm{E}$, depth $20 \mathrm{~m}$; SCUBA diving, don. D. Geiger, 28 Mar. 2006; T1616; NMSA-MOL 0W3498; Fig. 19 (Larger diameter: 0.74 mm).

Paratypes. South Africa - 2; same data as holotype; T1617; NMSAMOL 0W3499.

Current status. Scissurella maraisorum Geiger, 2006; original combination.

Remarks. The shell of the holotype is attached to the gelatine capsule and was not photographed to avoid possible damage. Visual examination confirms that it is the shell illustrated in the original description and larger diameter was measured using 


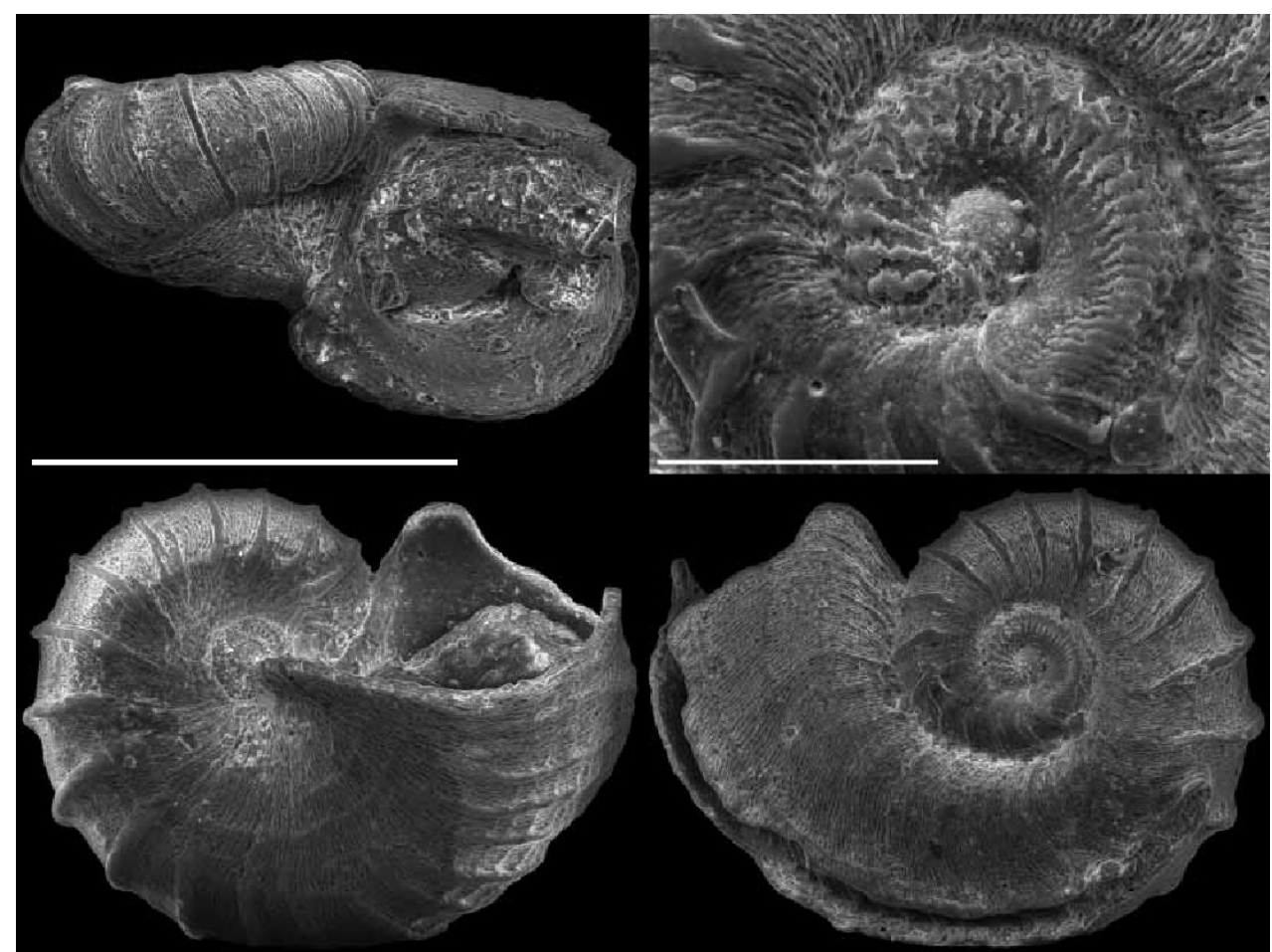

Figure 19. Scissurella maraisorum Geiger, 2006. Holotype T1616/NMSA-MOL 0W3498. Scale bars shell: $500 \mu \mathrm{m}$, protoconch: $100 \mu \mathrm{m}$. After Geiger (2006: 10, figure 7).

ocular micrometer, through the gelatine capsule. There are nine paratypes in other collections (see Geiger 2006: 10), all from the type locality. Coordinates were incorrectly rounded up in the original description.

\section{Sinezona doliolum Herbert, 1986}

doliolum Herbert, 1986: 625-626, figs 9, 28, 30 (holotype), 29, 31 (paratype) [Sinezona, off Mncwasa Point, Transkei $\left(32^{\circ} 06.2^{\prime} \mathrm{S}, 29^{\circ} 06.5^{\prime} \mathrm{E}\right), 68 \mathrm{~m}$, sand (dredged $\mathrm{MN})$ : diameter $0.9 \mathrm{~mm}$, height $1.0 \mathrm{~mm}$ ].

Material examined. Holotype. South Africa - Eastern Cape, off Mncwasa Point, $32^{\circ} 06.2^{\prime} \mathrm{S}, 29^{\circ} 06.5^{\prime} \mathrm{E}$, depth $68 \mathrm{~m}$, station N8; 19 Jul. 1982; dredged MN; sand; T3274; NMSA-MOL 0C8471; Fig. 20 (0.98×0.84×0.98 mm).

Paratypes. South Africa - Eastern Cape, off Ubombo, 31 55.3'S, 29²1.4'E, depth 96 m, station L6; 16 Jul. 1982; dredged MN; sand and gravel, T3275; NMSAMOL 0C7987; figured (figs 29, 31) in the original description. 

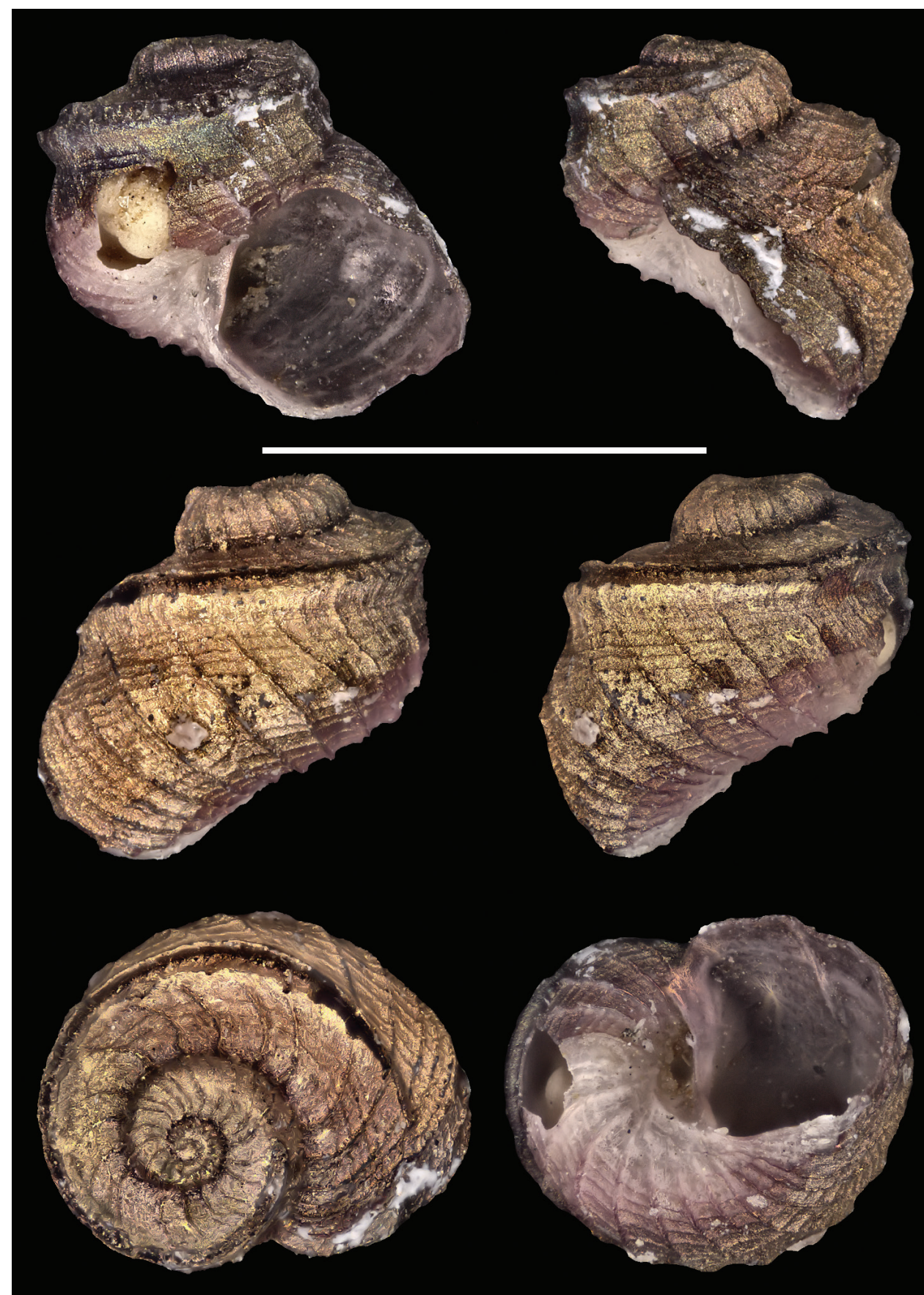

Figure 20. Sinezona doliolum Herbert, 1986. Holotype T3274/NMSA-MOL 0C8471. Scale bar: $1 \mathrm{~mm}$. 


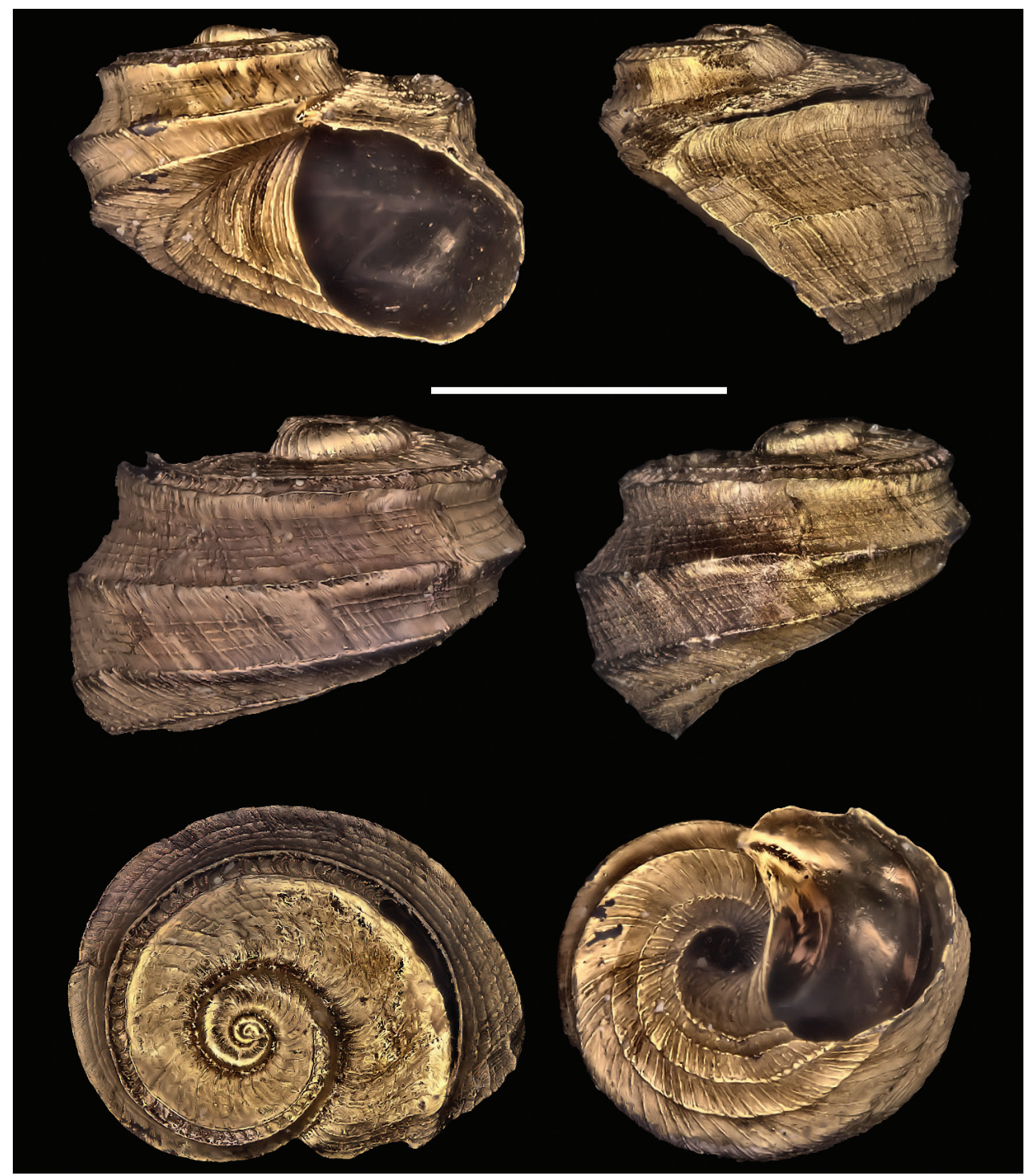

Figure 2I. Sukashitrochus maraisi Herbert, 1986. Holotype T3271/NMSA-MOL 0D684. Scale bar: 1 mm.

South Africa • same data as holotype; T3276; NMSA-MOL 0C8470.

South Africa • 6; Eastern Cape, off Mtamvuna River, 3108.6'S, 30²15.8'E, depth 106 m, station A11; 18 Aug. 1981; dredged MN some stones; T3277; NMSAMOL 0C8020.

Current status. Sinezona doliolum Herbert, 1986; original combination.

Remarks. The damage to the shell of the holotype was first discovered in March 2012 by the first author and the part that was broken off was not located. In the original description the damage cannot be assessed because of the position of the shell. Diameter in the figure captions 28 and 30 in the original description is $0.95 \mathrm{~mm}$. 


\section{Sukashitrochus maraisi Herbert, 1986}

maraisi Herbert, 1986: 629-631, figs 6, 37, 39 (holotype), 36, 38 (paratype) [Sukashitrochus, Mzamba, Transkei, beach drift (Marais 1976): diameter $1.5 \mathrm{~mm}$, height $1.1 \mathrm{~mm}$ ].

Material examined. Holotype. South Africa • Eastern Cape, Mzamba, beach drift; J.P. Marais leg.; Apr. 1976 (don. 6 May 1976; T3271; NMSA-MOL 0D0684; Fig. 21 $(1.48 \times 1.28 \times 1.07 \mathrm{~mm})$.

Paratypes. South Africa -2 same data as holotype; T3272; NMSA-MOL 0D0686.

South Africa 2 same data as holotype; T3273; NMSA-MOL 0D0685; figured (figs 36, 37) in the original description.

South Africa • 10; Eastern Cape, Mzamba, beach drift; 12-30 May 1986; D. Herbert and R. Kilburn leg.; T3368; NMSA-MOL 0D3483.

Current status. Sukashitrochus dorbignyi (Audouin, 1826); Herbert (2015).

Remarks. Diameter in the figure captions 37 and 39 in the original description is $1.45 \mathrm{~mm}$. Four paratypes mentioned in the original description collected at Mzamba, Eastern Cape are in the J. Marais collection and were not examined here.

\section{Acknowledgements}

We would like to thank Adam J. Baldinger for illustrations of type material deposited in The Museum of Comparative Zoology (Cambridge), Christine Zorn and Thomas von Rintelen for illustrations of type material deposited in the Museum für Naturkunde (Berlin), Linda Davis (NMSA) for the interpretation of some records on labels and in the catalogue books, John Midgley (NMSA) for his advice on the status on some type material, Andreia Salvador (Natural History Museum, London) for her valuable comments on the manuscript and David G. Herbert for his valuable comments on the status of some species and for the detailed review of the manuscript.

\section{References}

Adams H (1872) Further descriptions of new species of shells collected by Robert M'Andrew, Esq., in the Red Sea. Proceedings of the Zoological Society of London 40: 9-12. https:// www.biodiversitylibrary.org/page/28611355

Audouin V (1826) Explication sommaire des planches de mollusques de l'Egypte et de la Syrie publiées par J. C. Savigny. Description de l'Egypte ou recueil des observations et des recherches qui ont été faites en Egypte pendant l'expédition de l'armée française, publié par les ordres de sa majesté l'empereur Napoléon le grand. Histoire Naturelle, Animaux invertébrés. Imprimerie Impériale, Paris 1(4): 7-56. https://digi.ub.uni-heidelberg.de/diglit/ jomard1809bd5_1_1/0548 
Cunha TJ, Lemer S, Bouchet P, Kano Y, Giribet G (2019) Putting keyhole limpets on the map: Phylogeny and biogeography of the globally distributed marine family Fissurellidae (Vetigastropoda, Mollusca). Molecular Phylogenetics and Evolution 135: 249-269. https://doi. org/10.1016/j.ympev.2019.02.008

Geiger DL (2006) Eight new species of Scissurellidae and Anatomidae (Mollusca: Gastropoda: Vetigastropoda) from around the world, with discussion of two new senior synonyms. Zootaxa 1128(1): 1-33. https://doi.org/10.11646/zootaxa.1128.1.1

Geiger DL (2012) Monograph of the Little Slit-Shells. Santa Barbara Museum of Natural History, Santa Barbara, $1291 \mathrm{pp}$.

Geiger DL, Jansen P (2004) Revision of the Australian species of Anatomidae (Mollusca: Gastropoda: Vetigastropoda). Zootaxa 415(1): 1-35. https://doi.org/10.11646/zootaxa.415.1.1

Herbert DG (1986) A revision of the southern African Scissurellidae (Mollusca: Gastropoda: Prosobranchia). Annals of the Natal Museum 27(2): 601-632. https://hdl.handle. net/10520/AJA03040798_432

Herbert DG (1989) A remarkable new species of Diodora Gray, 1821 from south-east Africa (Mollusca: Gastropoda: Fissurellidae). Annals of the Natal Museum 30(1): 173-176. https://hdl.handle.net/10520/AJA03040798_353

Herbert DG (2015) An annotated catalogue and bibliography of the taxonomy, synonymy and distribution of the recent Vetigastropoda of South Africa (Mollusca). Zootaxa 4049(1): 1-98. https://doi.org/10.11646/zootaxa.4049.1.1

Herbert DG, Kilburn RN (1986) Taxonomic studies on the Emarginulinae (Mollusca: Gastropoda: Fissurellidae) of southern Africa and Mozambique. Emarginula, Emarginella, Puncturella, Fissurisepta and Rimula. South African Journal of Zoology 21(1): 1-27. https://doi.org/10.1080/02541858.1986.11447951

ICZN (1999) International code of zoological nomenclature ( $4^{\text {th }}$ Edn.). The International Trust for Zoological Nomenclature, London.

Kilburn RN (1977) Taxonomic studies on the marine Mollusca of southern Africa and Mozambique. Part 1. Annals of the Natal Museum 23(1): 173-214. https://hdl.handle.net/10520/ AJA03040798_574

Kilburn RN (1978) The Emarginulinae (Mollusca: Gastropoda: Fissurellidae) of southern Africa and Mozambique. Annals of the Natal Museum 23(2): 431-453.

McLean J, Geiger DL (1998) New genera and species having the Fissurisepta shell form, with generic-level phylogenetic analysis (Gastropoda: Fissurellidae). Contributions in Science 475: 1-32. https://www.biodiversitylibrary.org/page/52137375\#page/313/mode/1 up

McLean J, Kilburn RN (1986) Propodial elaboration in Southern African and Indian Ocean Fissurellidae (Mollusca: Prosobranchia) with description of two genera and one new species. Contributions in Science 379: 1-12. http://en.scientificcommons.org/52960182

Muratov IV (2014) Primary types in the collection of molluscs in the KwaZulu-Natal Museum: Polyplacophora. African Invertebrates 55(2): 377-412. https://doi.org/10.5733/ afin.055.0205

Muratov I, Davis L (2011) Primary types in the collection of molluscs in the KwaZulu-Natal Museum: Scaphopoda and Cephalopoda. African Invertebrates 52(2): 255-263. https:// doi.org/10.5733/afin.052.0203 
Nakano T, Ozawa T (2007) Worldwide phylogeography of limpets of the order Patellogastropoda: Molecular, morphological and palaeontological evidence. The Journal of Molluscan Studies 73(1): 79-99. https://doi.org/10.1093/mollus/eym001

Robson G (1986) A new species of South African Limpet, Patella aphanes, (Mollusca: Gastropoda: Patellidae), with a discussion of P. obtecta Krauss, 1848. Durban Museum Novitates 13(22): 305-320. https://hdl.handle.net/10520/AJA0012723X_2185

Smith EA (1901) On South African marine shells, with description of new species. Journal of Conchology 10: 104-116.

Smith EA (1906) On South African marine Mollusca, with descriptions of new species. Annals of the Natal Museum 1: 19-71.

Thiele J (1925) Gastropoda der Deutschen Tiefsee-Expedition. II Teil. In: Chun C (Ed.) Wissenschaftliche Ergebnisse Der Deutschen Tiefsee-Expedition Auf Dem Dampfer "Valdivia" 1898-1899. Gustav Fischer, 17, Jena, 35-382.

WoRMS Editorial Board (2020) World Register of Marine Species. http://www.marinespecies. org at VLIZ [accessed 2020-02-21] 